\title{
Integration of touch attention mechanisms to improve the robotic haptic exploration of surfaces
}

\author{
Ricardo Martins $^{\mathrm{a}, \mathrm{b}, *}$, João Filipe Ferreira ${ }^{\mathrm{a}}$, Miguel Castelo-Branco ${ }^{\mathrm{b}}$, Jorge Dias ${ }^{\mathrm{a}, \mathrm{c}}$ \\ a ISR-UC, Institute of Systems and Robotics, Department of Electrical and Computer Engineering - University of Coimbra Polo II, 3030-290 Coimbra, \\ Portugal \\ b IBILI-UC, Institute for Biomedical Imaging and Life Sciences, Faculty of Medicine, University of Coimbra, Coimbra, Portugal \\ ${ }^{\mathrm{c}}$ Robotics Institute, Khalifa University, Abu Dhabi, UAE
}

\section{A R T I C L E I N F O}

Communicated by Grana Manuel

Keywords:

Touch attention

Artificial perception

Bayesian modelling

Path planning

Haptic exploration

Probabilistic grid maps

\begin{abstract}
A B S T R A C T
This text presents the integration of touch attention mechanisms to improve the efficiency of the actionperception loop, typically involved in active haptic exploration tasks of surfaces by robotic hands. The progressive inference of regions of the workspace that should be probed by the robotic system uses information related with haptic saliency extracted from the perceived haptic stimulus map (exploitation) and a "curiosity"inducing prioritisation based on the reconstruction's inherent uncertainty and inhibition-of-return mechanisms (exploration), modulated by top-down influences stemming from current task objectives, updated at each exploration iteration. This work also extends the scope of the top-down modulation of information presented in a previous work, by integrating in the decision process the influence of shape cues of the current exploration path. The Bayesian framework proposed in this work was tested in a simulation environment. A scenario made of three different materials was explored autonomously by a robotic system. The experimental results show that the system was able to perform three different haptic discontinuity following tasks with a good structural accuracy, demonstrating the selectivity and generalization capability of the attention mechanisms. These experiments confirmed the fundamental contribution of the haptic saliency cues to the success and accuracy of the execution of the tasks.
\end{abstract}

\section{Introduction}

In an attempt to capitalise on the same advantages that having hands benefit human beings, researchers have recently put a lot of effort into the development of dexterous robotic hands, due to the mechanical (high number of degrees-of-freedom) and sensory (tactile, force, torque, heat) capabilities that they provide. These devices allow robotic platforms to perform precise manipulation of objects (reaching, grasping, transportation, in-hand reorientation) [1], as well as haptic exploration of surfaces using different patterns of movements (lateral motion, press-and-release, static contact), thereby promoting the extraction and integration of different haptic properties (contours, texture, compliance, temperature) of the materials these surfaces are composed of [2].

The contributions presented in this work are related with the robotic haptic exploration of surfaces, following three essential assumptions: (1) no other type of sensors are used besides haptics (i.e. exploration is "blind"); (2) exploration paths are not predefined; (3) the surface geometry is unknown to the robot. The objectives of the exploration tasks concern haptic discontinuity/contour following. Haptic discontinuities are defined by the transition/border regions between surfaces with different haptic properties. During haptic exploration, the interaction of the robotic platform with the probed surface provides multiple simultaneous streams of data over its geometry and the properties of its composing materials relayed by an ensemble of haptic sensors. This data is potentially uncertain due to sensor noise and the unknown nature of the surface.

To tackle these challenges, we propose a Bayesian framework to implement autonomous haptic exploration of surfaces that implements an action-perception loop architecture. The Bayesian formalism provides a principled way of implementing the integration of the multimodal sensory data supplied by the haptics ensemble while properly dealing with their inherent uncertainty. The proposed action-perception loop architecture integrates touch attention mechanisms (i.e. stimulus-driven processes modulated by task-relevant top-down influences) to optimise the exploration strategy. This in turn promotes

\footnotetext{
* Corresponding author at: University of Coimbra Polo II, Department of Electrical and Computer Engineering, ISR-UC, Institute of Systems and Robotics, 3030-290 Coimbra, Portugal.

E-mail addresses: rmartins@isr.uc.pt (R. Martins), jfilipe@isr.uc.pt (J.F. Ferreira), mcbranco@fmed.uc.pt (M. Castelo-Branco), jorge@isr.uc.pt (J. Dias).
} 
adaptive behaviour due to the exploration and exploitation traits of such mechanisms.

The haptic exploration of surfaces plays a fundamental role in reduced visibility scenarios (i.e.: underwater robotic manipulation, smoky and foggy disaster environments, partial or complete occlusion of elements in the scenario). Although this work only addresses the implementation of haptic exploration strategies, the proposed Bayesian framework allows the integration of additional sensory sources such as vision (depth, color) and laser to infer the robotic exploration path. The approach proposed in this work can be used to complement methods already available to explore surfaces using exclusively non-haptic sensory inputs [4-6].

The structure of the manuscript and an overview of the Bayesian models proposed in this work are presented in Section 1.1.

\subsection{Problem formulation and approach overview}

In the application scenarios used in this work, the exploration task is performed on top of a table - a workspace defined by a planar surface - and using a generic robotic system with manipulation capability. The internal structure and configuration of the workspace is unknown a priori to the robotic system. The solution to the haptic exploration task is described in two-dimensional Cartesian space, progressively unfolding a sequence of regions of the workspace to be probed by the robotic platform during task execution.

As in previous reported work, the 2D-Cartesian space is partitioned using a planar isometric 2D grid (square cells), as represented in Fig. 1 b). Each cell $v_{k}$ has a side of length $\varepsilon$ and is described by a 2D Cartesian location $(x, y)$ expressed in the inertial world referential $\{\mathcal{W}\}$. These tesselations of space have been used extensively in robotics as inference grids in many applications [9].

The methods presented follow the principles and architecture of the human somatosensory processing pipeline and human cognition. A conceptual overview of our solution is presented in Fig. 2; the corresponding detailed diagram is given in Fig. 3, including a representation of data flow. Haptic sensory inputs are acquired during the local interaction of the robotic exploratory elements with the environment at region $\mathbf{v}_{k}$. Haptic features such as texture, compliance, temperature are extracted from the haptic sensory inputs. These features are integrated and used to discriminate the different classes of materials in the workspace. These processes are modelled by the Bayesian model $\pi_{\text {per }}$ presented in Section 3.

Next in the sensory processing pipeline, the robotic system uses the updated perceptual representation of the workspace to infer the next region that should be explored. The mechanisms involved in this process are implemented by the Bayesian model $\pi_{\text {tar }}$ and described in Section 5. Touch attention is modelled by integrating the following:

- Stimulus-driven processes - concurrent mechanisms that pro-

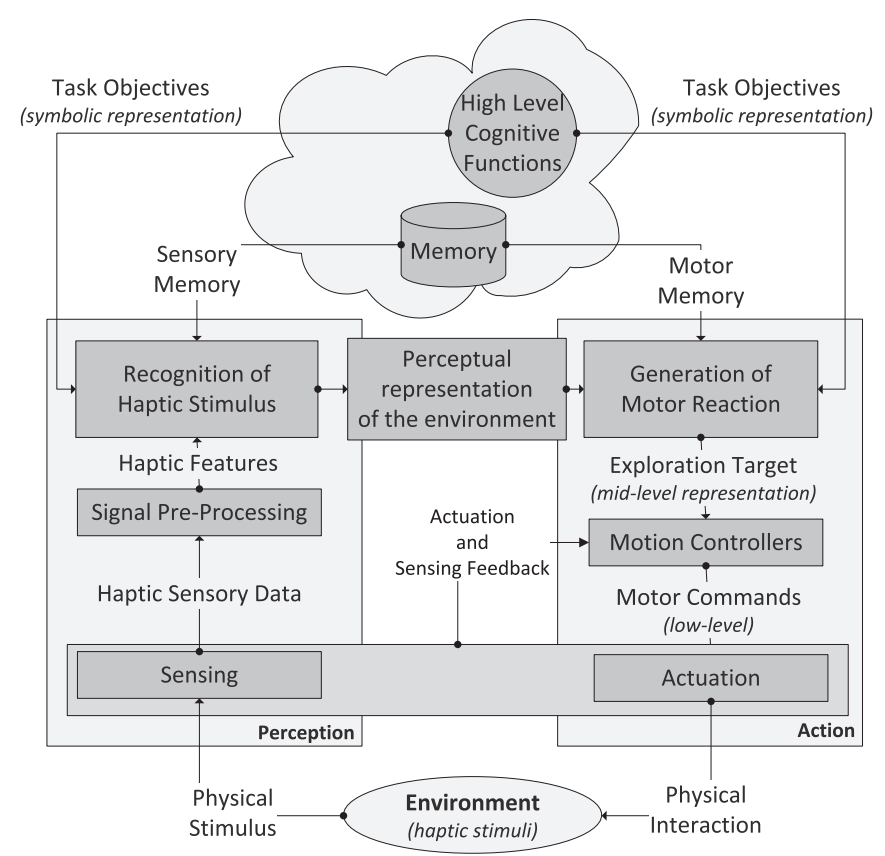

Fig. 2. Conceptual representation of the action-perception loop [7] involved in the haptic exploration of surfaces [8]. In this work, the objectives of the task and corresponding solution is represented in two levels: symbolic and mid-level.

mote both exploitation behaviour concerning perceptual representations of stimuli in the form of haptic saliency and shape cues (determined by the Bayesian model $\pi_{o b j}$, Section 4), and exploration behaviours fuelled by spatial distribution of perceptual uncertainty and also inhibition-of-return mechanisms.

- Goal-directed modulation - mechanisms that influence the weights of stimulus-driven processes through top-down influences informed by current task objectives.

The experimental setup used in this work is described in Section 6 . The impact of the integration of the touch attention mechanisms in the action-perception loop and generalization capability of the exploration strategies inferred from the proposed Bayesian models are tested in simulation environment, Section 6. The main conclusions of this work and formulation of the main guidelines for future developments of this approach are presented in Section 7.

\subsection{Path planning of the global haptic exploration strategy}

The framework conceptually represented in Fig. 2 and detailed in Fig. 3 implements a haptic exploration path planning method, which infers a series of global via-points in the workspace that should be

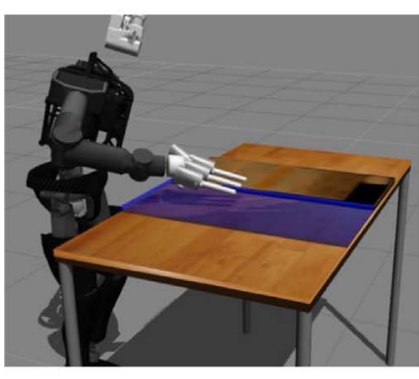

(a)

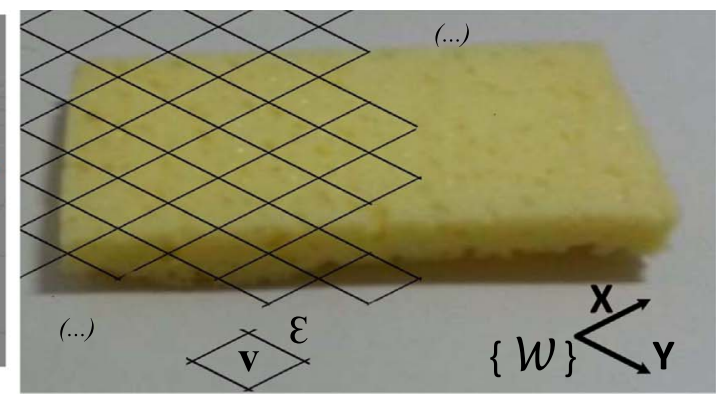

(b)

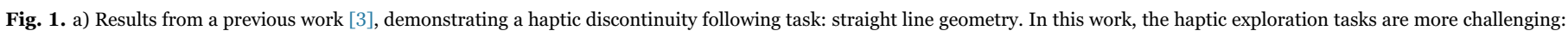

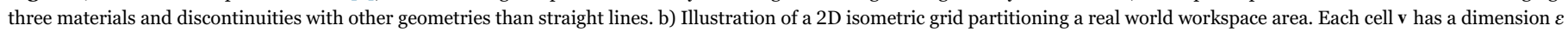
and is described by position $(x, y)$ expressed in $\{\mathcal{W}\}$. 


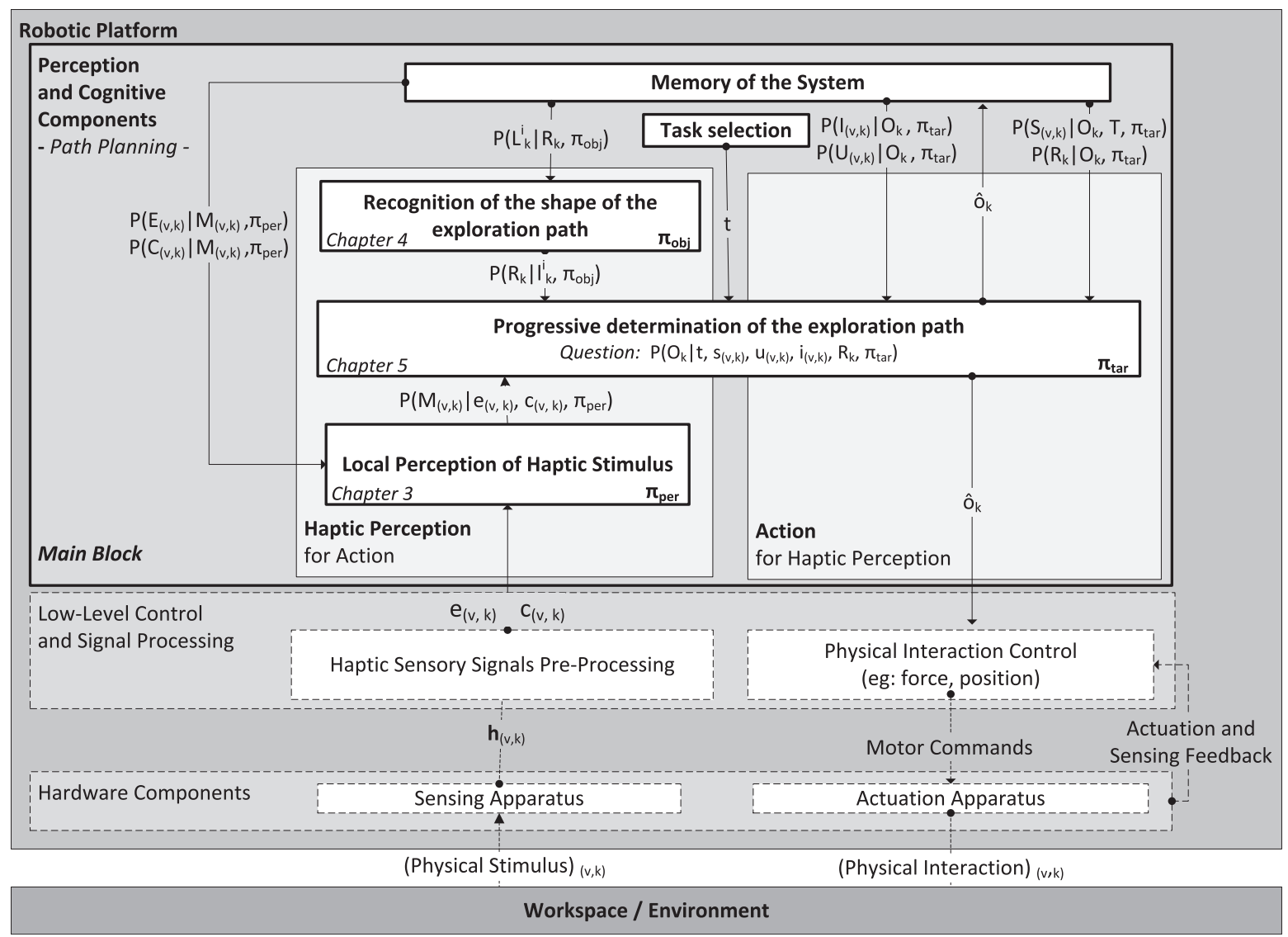

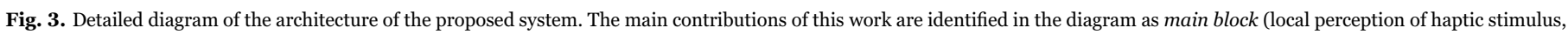
recognition of the shapes of discontinuities, progressive determination of the exploration path). The variables of the system are summarised in Table 1 .

probed by the robotic system.

This work does not address the low-level control loop involved in physical interaction of the fingers with the surface and the ability to move the fingers along the surface by keeping contact. In other words, the low-level modelling and control of local contact interaction (eg: force, impedance, position control) and processing of haptic sensory data are not discussed by this work. These processes are implemented in Fig. 3 by the module Low-Level Control and Signal Processing and inner loop labelled Actuation and Sensing Feedback.

Our solution assumes that algorithms (dependent of specific robotic device and sensing apparatus) implemented by other works (eg: [10]) extract different haptic features and control the local movements during the haptic exploration of a region $\mathbf{v}_{k}$. The integration between these lower level control models (dashed boxes) and the global exploration path planning method (bold boxes) proposed by this work is detailed in Fig. 3.

\section{Related works}

The robotic exploration of surfaces using haptic inputs has been a research topic pursued for a long time, with seminal works by [23-25] and [26].

A group of approaches described in the literature implements haptic exploration by attempting to achieve a single categorisation of surfaces or objects (Table 2). The exploration is performed locally in a specific region, considering that it is representative of the whole surface by assuming that the latter is either homogeneous or uniform in terms of the haptic features under analysis. The discrimination between the different classes of surfaces is performed by extracting distinct but complementary types of haptic features such as surface curvature [11], texture $[12,10,13,14]$, compliance $[10,15]$, stickiness [16] and thermal conductivity $[10,17]$ from the haptic sensory signals. The formalisation of the descriptors of the haptic features depends on the type of robotic platform and type of sensing apparatus involved in the exploration task, specifically the modelling of the contact interaction and the characteristics of the sensory signals produced during that interaction. However, each type of haptic feature is extracted using the same exploration movement patterns across the different works.

The work presented in this manuscript contributes to this group of approaches by introducing a Bayesian model that allows the discrimination different categories of materials through the integration of compliance and texture features. The formulation of haptic features abstracts the contact interaction models between the exploratory element and the surface.

A second group of approaches (Table 2), while integrating sensing, perception and local exploration mechanisms similarly to the previous group, expands the exploration strategy to large and heterogenous surfaces in the haptic feature domain under analysis. The global perceptual map of the surface can be constructed following different strategies. In many proposed solutions, the global exploration path is fixed and defined a-priori. For example, in Ref. [18], haptic exploration is performed using pre-defined exploration paths to build a stiffness map of biological tissues. As long as the perception of the haptic stimulus of the surface occurs, it does not influence the exploration movement. In Ref. [19], Braille symbols are explored and recognised by a robotic system. The exploration speed is adjusted depending on the recognition uncertainty, nevertheless the exploration path is also predefined.

Although exploration strategies defined a-priori can be successful when substantial information about the structure of the environment is available, in most of the scenarios identified considering the motivation behind this work, the structure of the environment is initially unknown 
Table 2

Comparison between the contributions of this work and the related works.

\begin{tabular}{|c|c|c|c|c|c|c|c|}
\hline \multirow[t]{2}{*}{ Study } & \multirow[t]{2}{*}{ Apparatus $^{\mathrm{a}}$} & \multicolumn{2}{|c|}{ Local Haptic Perception } & \multicolumn{4}{|c|}{ Global Exploration of the workspace } \\
\hline & & Approach $^{\mathrm{b}}$ & Features $^{c}$ & Approach $^{\mathrm{d}}$ & Task $^{\mathrm{e}}$ & Strategy $^{\mathrm{f}}$ & Workspace $^{\mathrm{g}}$ \\
\hline This Work & HS & $\mathrm{P}$ & $\mathrm{T}, \mathrm{CO}$ & $\mathrm{P}$ & M: E, F: E & $\mathrm{AE}$ & GD:2D \\
\hline [11] & HS & $\mathrm{D}$ & $\mathrm{C}$ & - & - & - & - \\
\hline$[12]$ & HS & $\mathrm{D}$ & $\mathrm{T}$ & - & - & - & - \\
\hline [10] & HS & $\mathrm{P}$ & $\mathrm{CO}, \mathrm{T}, \mathrm{TC}$ & - & - & - & - \\
\hline [13] & HS & $\mathrm{P}$ & $\mathrm{T}$ & - & - & - & - \\
\hline [14] & HS & $\mathrm{D}$ & $\mathrm{T}$ & - & - & - & - \\
\hline$[15]$ & HS & $\mathrm{P}$ & $\mathrm{C}$ & - & - & - & - \\
\hline$[16]$ & HS & $P$ & $\mathrm{~S}$ & - & - & - & - \\
\hline [17] & HS & $\mathrm{D}$ & $\mathrm{C}, \mathrm{TC}$ & - & - & - & - \\
\hline [18] & HS & $\mathrm{D}$ & $\mathrm{CO}$ & $\mathrm{D}$ & M: C & PD & CS:2D \\
\hline [19] & HS & $\mathrm{D}$ & RO & $\mathrm{P}$ & $\mathrm{F}: \mathrm{T}$ & PD & - \\
\hline [20] & HS & $\mathrm{P}$ & $\mathrm{C}$ & $\mathrm{D}$ & $\mathrm{F}: \mathrm{C}$ & $\mathrm{AE}$ & - \\
\hline [21] & HS, VS & $\mathrm{P}$ & TO & $\mathrm{P}$ & M: E & $\mathrm{AE}$ & GD:2D \\
\hline [22] & $\mathrm{HS}$ & $\mathrm{P}$ & RO & $\mathrm{D}$ & M: E, F: E & $\mathrm{AE}$ & GD:2D \\
\hline [20] & HS & $\mathrm{D}$ & CI, CR & $\mathrm{D}$ & $\mathrm{F}$ & $\mathrm{AE}$ & $\mathrm{CS}: 3 \mathrm{D}$ \\
\hline
\end{tabular}

${ }^{a}$ HS- haptic sensing; VS- visual sensing.

${ }^{\mathrm{b}} \mathrm{P}$ - probabilistic; D- deterministic.

${ }^{\mathrm{c}} \mathrm{T}$ - texture; CO- compliance; C- curvature; TC- thermal conductivity; S- stickiness; RO- raw sensory output; CI- contact intensity; CR- contact orientation.

${ }^{\mathrm{d}} \mathrm{P}$ - probabilistic; D- deterministic.

e M: E- mapping edge; M: C - mapping compliance; F- following; F: E- following edge; F: T- following texture; F:C: following curvature.

${ }^{\mathrm{f}}$ AC- active exploration; PD- pre-defined exploration path.

${ }^{\mathrm{g}}$ GD:2D - bi-dimensional grid; CS:2D- bi-dimensional Cartesian space points; CS:3D- tri-dimensional Cartesian space points.

(partially or completely). Thus, the exploration strategy should introduce an active behaviour to progressively integrate and analyse the local perceptual representation of the environment (perception for action) and estimate what should be the next global region to explore and perceive (action for perception), as proposed in Ref. [27]. Active exploration of a scene represented by a occupancy grid was proposed by [21]. An initial estimation of the scene structure is made using stereovision data and projected in a 2D occupancy grid. The exploration strategy is dependent of that initial representation and haptic inputs (lateral contact/non-contact) are used to confirm and update the occupancy grid of the map.

In other works, the active exploration task is started without any knowledge about scene structure. For example, [22] proposes a method to perform active contour following of objects by performing tap movements using a robotic fingertip equipped with a tactile array. The reaction of the system is formulated based on the contact profile between the haptic stimulus and the tactile sensing array and specific deterministic rules defined beforehand by the human operator. [20] presents a generic formulation of a control framework for different types of tasks that require tactile servoing (eg: tracking a touched object, tactile object active exploration). Different behaviours are obtained by adjusting a few matrix parameters and selecting the corresponding haptic primitives extracted from a tactile array.

This work adds to the contributions of this class of approaches by proposing a formulation of Bayesian models implementing touch attention mechanisms involved in the active haptic exploration of unknown surfaces by generic robotic hands and sensory apparatus. Once this work assumes that the workspace is unknown a-priori to the system (blind exploration), the exploration path is adapted actively by the touch attention mechanisms, as long as the exploration progresses. The definition of the architecture of the Bayesian models follows the principles on how humans manage uncertainty to make motor decisions from percepts [7], and extends the architecture proposed in a previous work [3]. The work presented in this manuscript expands the top-down modulation of information, by integrating an additional Bayesian model in the decision process, representing the influence of the shape of the current exploration path (detailed in Section 4). In Ref. [3] the experimental results were focused on testing extensively the capability of the system to discriminate different type of materials. In the current work, a completely new set of experiments is designed to test the selectivity (different types of discontinuities) and robustness (different path shapes) of the touch attention mechanisms during three haptic discontinuity following tasks. The new experimental design was also used to evaluate the contributions of the different types of cues modelled by the Bayesian models to the performance of the robotic system.

\section{Local perception of haptic stimulus map}

\subsection{Random variables of the model}

The type of material describing workspace region $v$ is represented by the discrete random variable $M_{(v, k)}$, defined as follows:

$M_{(v, k)} \in\left\{\right.$ Material $_{1}, \ldots$, Material $\left._{10}\right\}$.

During local exploration of region $v$ of the workspace at time iteration step $k$, the robotic system acquires haptic sensory data represented by variable $\mathbf{h}_{(v, k)}$. The categories of materials are discriminated according to different properties of texture and compliance, hence haptic sensory inputs $\mathbf{h}_{(v, k)}$ are used to determine the category of material describing the cell $v$ of the workspace. Haptic sensing measurements $\mathbf{h}_{(v, k)}$ are transformed using function $g$ into a compliance characterization of the explored surface, and using function $f$ into a texture characterization of the surface. This work considers the same operator functions $f$ and $g$ of the work [10]. The texture and compliance characteristics of the region $v$ of the workspace are described by the continuous random variables, $E_{(v, k)} \equiv$ "Texture characterization of $v$ ", and $C_{(v, k)} \equiv$ "Compliance characterization of v", respectively, according to the following expressions:

$E_{(v, k)}=f\left(\mathbf{h}_{(v, k)}\right), \quad E_{(v, k)} \in \mathbb{R}, C_{(v, k)}=g\left(\mathbf{h}_{(v, k)}\right), \quad C_{(v, k)} \in \mathbb{R}$.

\subsection{Inference of the haptic stimulus category}

The Bayesian model $\pi_{p e r}$ allowing the estimation of surface material given haptic sensory inputs (Fig. 4) was extensively tested in previous work [3], in which it was used to discriminate between different classes of materials (the same set of 10 different classes used in the work presented in Ref. [10], more specifically acrylic, brick, 


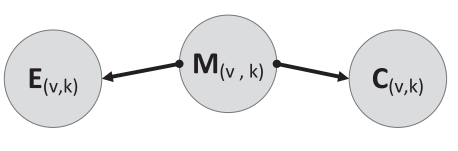

(a)

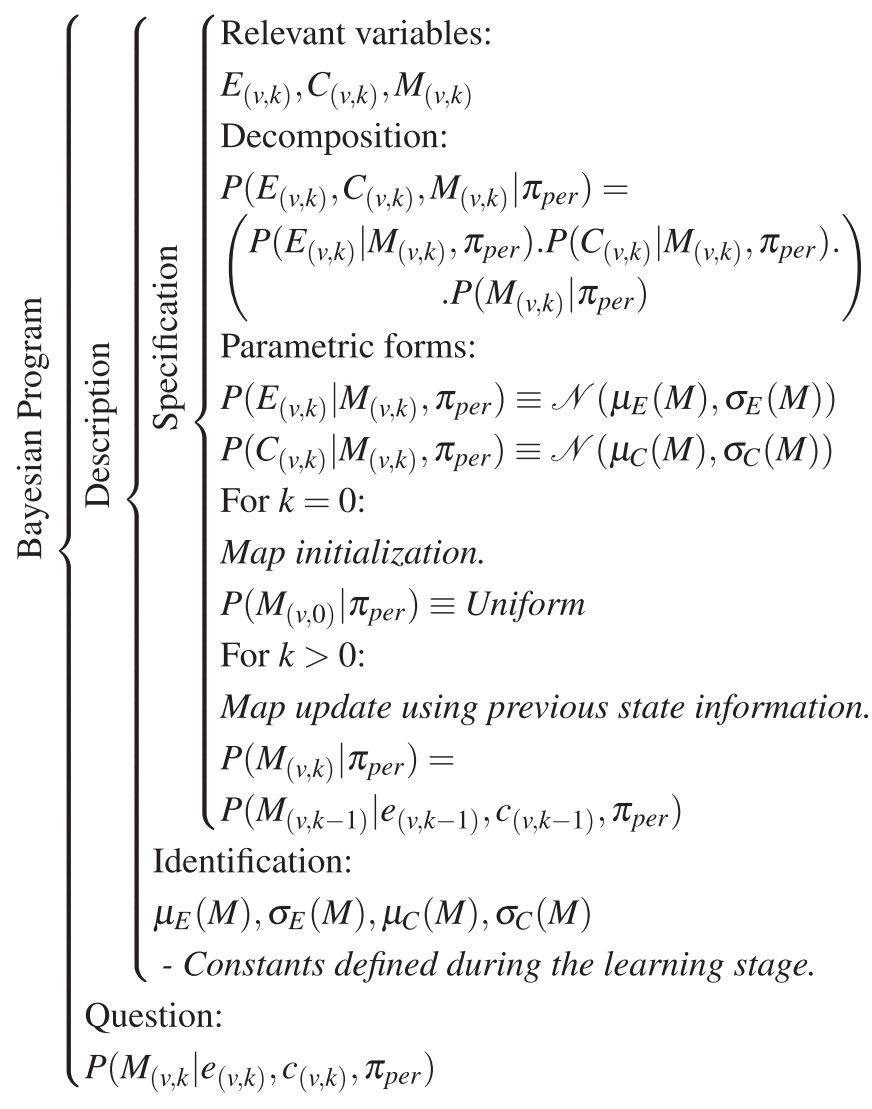

(b)

Fig. 4. Bayesian model $\pi_{\text {per: }}$ "Local perception of haptic stimulus". a) Graphical representation. b) Description of the Bayesian program.

copper, damp sponge, feather, rough foam, plush toy, silicone, soft foam, wood) with an average recognition rate higher than $90 \%$, even when sensory samples were corrupted with Gaussian white noise. These categories of materials are characterised by different properties of texture, compliance and thermal conductivity that were extracted using BioTac biomimetic tactile sensor raw data (contact intensity, vibration, heat flow). In our work, we only consider texture and compliance properties of the materials.

The conditional independence relations between random variables $E_{(v, k)}, C_{(v, k)}, M_{(v, k)}$ are expressed in Fig. 4a). Based on these assumptions, the joint probability distribution function $P\left(E_{(v, k)}, C_{(v, k)}, M_{(v, k)} \mid \pi_{p e r}\right)$ is decomposed as described in Fig. 4b), with respective parametric forms.

At each time step, the probability distribution function $P\left(M_{(v, k)} \mid e_{(v, k)}, c_{(v, k)}, \pi_{p e r}\right)$ describing the probability of the surface at $v$ corresponding to each material category is inferred using the observed data $e_{(v, k)}, c_{(v, k)}$ extracted from the samples acquired by the sensory apparatus of the robotic system:

$P\left(M_{(v, k)} \mid e_{(v, k)}, c_{(v, k)}, \pi_{p e r}\right)=\frac{\left(\begin{array}{c}P\left(e_{(v, k)} \mid M_{(v, k)}, \pi_{p e r}\right) . \\ P\left(c_{(v, k)} \mid M_{(v, k)}, \pi_{p e r}\right) . P\left(M_{(v, k)}, \pi_{p e r}\right)\end{array}\right)}{\sum_{M_{(v, k)}}\left(\begin{array}{c}P\left(e_{(v, k)} \mid M_{(v, k)}, \pi_{p e r}\right) . \\ P\left(c_{(v, k)} \mid M_{(v, k)}, \pi_{p e r}\right) . P\left(M_{(v, k)}, \pi_{p e r}\right)\end{array}\right)}$

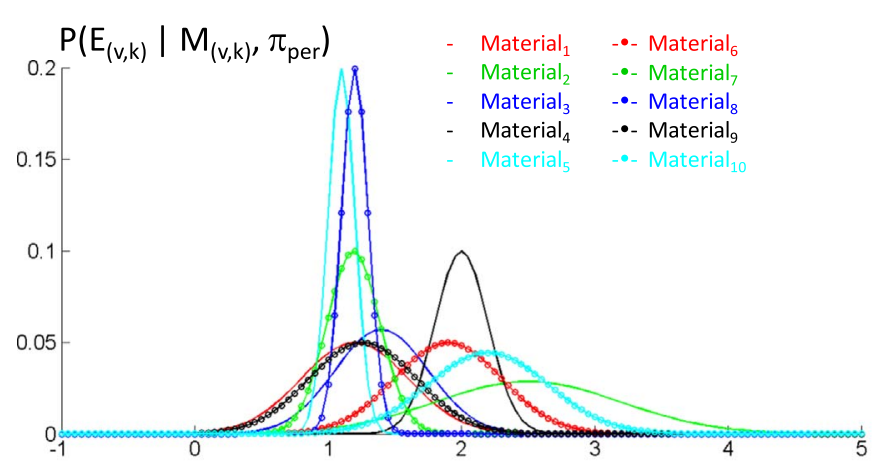

(a)

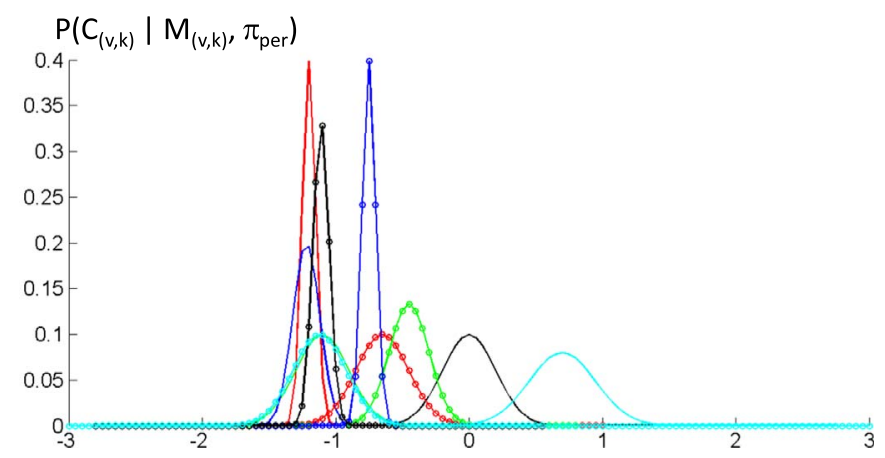

(b)

Fig. 5. Representation of $\left.P\left(E_{(v i, k)} \mid M_{(v i, k)}, \pi_{p e r}\right)(\mathrm{a})\right)$ and $P\left(C_{(v i, k)} \mid M_{(v i, k)}, \pi_{p e r}\right)$ (b)) learned for 10 reference materials. Data extracted from [10].

\subsection{Determination of $P\left(E_{(v, k)} \mid M_{(v, k)}, \pi_{p e r}\right)$ and $P\left(C_{(v, k)} \mid M_{(v, k)}, \pi_{p e r}\right)$}

The parameters $\mu_{E}(M), \sigma_{E}(M), \mu_{C}(M), \sigma_{C}(M)$ of the Gaussian functions modelling the Normal probability distributions $P\left(E_{(v, k)} \mid M_{(v, k)}, \pi_{p e r}\right)$ and $P\left(C_{(v, k)} \mid M_{(v, k)}, \pi_{p e r}\right)$ are estimated during experimental learning sessions using a maximum-likelihood procedure. As described in Ref. [10], during the learning period, standard local exploration procedures are performed for each of the $n=10$ reference materials.

After the pre-determined number of standard local explorations, the free parameters $\mu_{E}(M), \sigma_{E}(M), \mu_{C}(M), \sigma_{C}(M)$ of the Normal $(\mathcal{N})$ distributions are determined by calculating the averages $\mu$ and standard deviations $\sigma$ of $E$ and $C$ for each reference material. The resulting $P\left(E_{(v, k)} \mid M_{(v, k)}, \pi_{p e r}\right)$ and $P\left(C_{(v, k)} \mid M_{(v, k)}, \pi_{p e r}\right)$ are represented in Fig. 5 a) and b), extracting the data available from the manuscript of the work [10].

\section{Recognition of the shape of the global exploration path}

\subsection{Random variables of the model}

As the haptic exploration of the workspace progresses, the exploration path is described by the set of regions of the two-dimensional workspace grid probed by the robotic system. The shape of the exploration path provides cues that can be recognised by the haptic exploration framework.

The category of the shape of the exploration path is represented by discrete random variable $R_{k}$, defined as follows:

$R_{k} \in\left\{\right.$ Shape $_{1}, \ldots$, Shape $\left._{\Theta}\right\}$.

Each class of shape described by discrete random variable $R_{k}$ is associated with a template, represented by a set of points template ${ }_{i}$,

$\forall_{i \in\{1, \ldots, \Theta\}} \quad\left\langle\right.$ "Shape $i$ ", template $\left._{i}\right\rangle$.

This work assumes that the robotic system is able to recognize 
$\Theta=2$ categories of shapes: a rectangle and a triangle, respectively.

The sequence of regions of the workspace explored by the robotic system until time step $(k-1)$ is described by the set of workspace locations $\left(\widehat{o}_{0}, \widehat{o}_{1}, \ldots, \widehat{o}_{k-1}\right)$ (Section 5$)$. The categorisation process consists of establishing a match between the points $\left(\hat{o}_{0}, \widehat{o}_{1}, \ldots, \widehat{o}_{k-1}\right)$ explored by the robotic system until time step $(k-1)$ and each of the

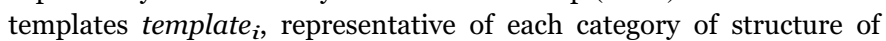
discontinuity. The normalised matching error between each template and the current exploration path is described by the continuous random variable $L^{i}$, defined as follows:

$\left[L^{i}, Y_{i}\right]=f_{I C P}\left(\left(\hat{o}_{0}, \hat{o}_{1}, \ldots, \hat{o}_{k-1}\right)\right.$, template $\left._{i}\right), \quad L^{i} \in[0,1]$

The matching between the two sets of points $\left(\widehat{o}_{0}, \widehat{o}_{1}, \ldots, \widehat{o}_{k-1}\right)$ and template $_{i}$ is determined using the Iterative Closest Point (ICP) method [28], as described in Eq. (6).

Besides matching error $L^{i}$, the ICP function $f_{I C P}$ returns the estimation of the geometrical transformation $Y_{i}$ between the two sets of points. This transformation can be used to determine a new set of points template $_{i}^{\prime}$ which results from the registration of the template $e_{i}$ points in the structure described by the set of points $\left(\widehat{o}_{0}, \widehat{o}_{1}, \ldots, \widehat{o}_{k-1}\right)$.

This relation can be described by the geometrical transformation represented in the following equation,

template $_{i}^{\prime}=Y_{i}$. template $_{i}$,

as used in Section 5.

\subsection{Inference of category of shape}

The graphical representation of Bayesian model $\pi_{o b j}$ presented in Fig. 6 a) expresses the conditional independence relations between random variables $L_{k}^{1}, \ldots, L_{k}^{\Theta}, R_{k}$. According to these relations, the joint probability distribution function $P\left(L_{k}^{1}, \ldots, L_{k}^{\Theta}, R_{k} \mid \pi_{o b j}\right)$ can be factored as presented in Fig. 6 b). The probability distribution function followed by each of those factors is also presented in Fig. 6 b).

At each time step, the probability distribution function $P\left(R_{k} \mid l_{k}^{1}, \ldots, l_{k}^{\Theta}, \pi_{o b j}\right)$ is inferred using the Bayesian program of Fig. 6

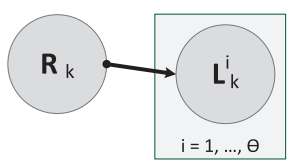

(a)

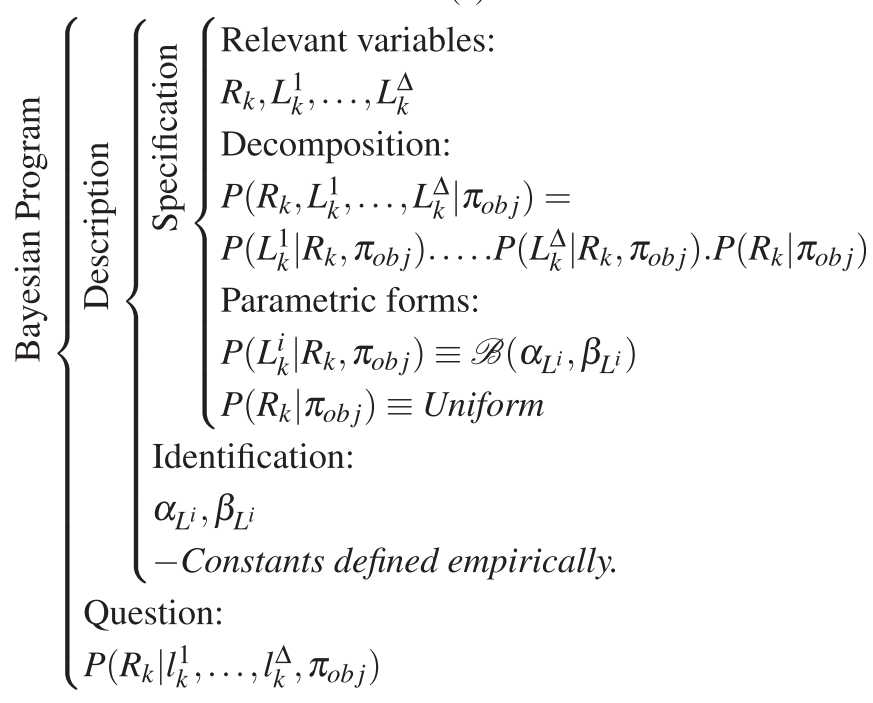

Fig. 6. Bayesian model $\pi_{o b j}$ : "Recognition of the shapes of discontinuities.". a) Graphical representation. b) Description of the Bayesian program. through the following equation:

$P\left(R_{k} \mid l_{k}^{1}, \ldots, l_{k}^{\Theta}, \pi_{o b j}\right)=\frac{P\left(l_{k}^{1} \mid R_{k}, \pi_{o b j}\right) . \ldots P\left(l_{k}^{\Theta} \mid R_{k}, \pi_{o b j}\right) . P\left(R_{k} \mid \pi_{o b j}\right)}{\sum_{R_{k}} P\left(l_{k}^{1} \mid R_{k}, \pi_{o b j}\right) \ldots P\left(l_{k}^{\Theta} \mid R_{k}, \pi_{o b j}\right) P\left(R_{k} \mid \pi_{o b j}\right)}$

\subsection{Determination of $P\left(l_{k}^{i} \mid R_{k}, \pi_{o b j}\right)$}

The probability distribution functions $P\left(l_{k}^{i} \mid R_{k}, \pi_{o b j}\right)$ are described by beta probability distribution functions $\mathcal{B}_{L}$ with the constant parameters $\alpha_{L}=1.0$ and $\beta_{L}=4.5$. All $\Theta$ probability distribution functions are assumed identical.

The typical profile of the probability distribution function $P\left(l_{k}^{i} \mid R_{k}, \pi_{o b j}\right)$ is represented in Fig. 8b). The profile proposed for $P\left(l_{k}^{i} \mid R_{k}, \pi_{o b j}\right)$ attributes higher probabilities to lower levels of normalised matching errors $l_{k}^{i}$ and lower probabilities to higher values of $l_{k}^{i}$. This promotes the selection of categories of the structure $R_{k}$ that have a template similar to the current exploration path $\left(\hat{o}_{0}, \hat{o}_{1}, \ldots, \widehat{o}_{k-1}\right)$.

\section{Integration of touch attention mechanisms in the inference of the global exploration path}

\subsection{Random variables of the model}

After the local exploration of the region $v$ of the workspace is concluded, the perceptual representation of the workspace is updated with the sensory measurements acquired at $v$ (update mechanisms presented in Section 3), and the robotic system has to decide which region $v$ of the workspace grid should be explored next (path planning of global exploration strategy).

The next exploration target is represented by the discrete random variable $O_{k}$, defined as

$O_{k} \in\left\{v^{1}, v^{2}, v^{3}, \ldots, v^{\theta}\right\}$,

in which $\theta$ is the total number of cells in the grid representation of the workspace, and $v^{i}$ is a compact representation of the cell identifier.

Robotic platforms have been endowed with attentional mechanisms implemented in different sensory domains in order to deal with sensory overload, prioritisation and dynamic environments [29].

The sequence of workspace regions $\left(\widehat{o}_{0}, \widehat{o}_{1}, \ldots, \widehat{o}_{k-1}\right)$ previously explored by robotic system, can provide cues about the shape of the discontinuity that is being followed and indirectly influence the estimation of $\widehat{o_{k}}$. The cues are provided by matching the current structure of the exploration path, with representations of typical shapes stored in the memory of the robotic system. As presented in Section 5.3 , unexplored regions of the workspace that are coincident with the structure of the shape templates will be more likely to be explored.

The selection of $O_{k}$ is also conditioned by inhibition-of-return mechanisms. The inhibition level imposed this mechanism to the overall attention process is implemented by the continuous random variable $I_{(v, k)}=$ "Inhibition level for cell $v$ " as follows:

$I_{(v, k)}=1-\Theta d^{\alpha-1}(1-d)^{1-\beta}, \quad I_{(v, k)} \in[0,1]$

Due to the characteristics of the haptic exploration procedures presented in Section 1, at time step $k+1$ the inhibition-of-return process promotes the exploration of regions of the workspace different from the current position of the end-effector of the robotic system $\left(\widehat{o}_{k-1}\right)$, therefore avoiding deadlocks. However, simultaneously, the inhibition-of-return process inhibits the exploration of regions too distant from $\hat{o}_{k-1}$, to avoid breaks during the discontinuity following task. The inhibition levels $I_{(v, k)}$ for each cell $v$ are defined in Eq. (10), considering $\alpha=1.01$ and $\beta=9$ (corresponding plot presented in Fig. 8 (a)). Parameter $d$ is given by $d=d_{k} / d_{\max }$, where $d_{k}$ expresses the Euclidean distance between $o_{k}$ and $\widehat{o}_{k-1}$, and $d_{\max }$ is a constant representing the maximum possible distance between $o_{k}$ and $\widehat{o}_{k-1}$ for 
the workspace dimensions. $\Theta$ is a normalisation constant. The values of $I_{(v, k)}(d)$ range from 0 to 1 , with $I_{(v, k)}=0$ indicating that the inhibitionof-return mechanism applies no inhibition to cell $v$, and $I_{(v, k)}=1$ signalling full inhibition of cell $v$.

The selection of the region $O_{k}$ of the workspace is also dependent on mechanisms that prevent returning to regions already explored and perceived with low uncertainty. In a nutshell, these mechanisms are formulated to promote the "curiosity", and are represented by the continuous random variable $U_{(v, k)}=$ "Uncertainty level for cell v", described as

$U_{(v, k)}=\frac{\mathcal{H}\left(M_{(v, k)}\right)}{\max \left(\mathcal{H}\left(M_{(v, k)}\right)\right)}, \quad U_{(v, k)} \in[0,1]$,

in which the operator $\mathcal{H}$ determines the information entropy [30] of the discrete random variable $M_{(v, k)}$.

Another factor conditioning the determination of $O_{k}$ is the saliency of the haptic stimulus for region $v$ as comparing to its surroundings. Besides depending on the perceived haptic stimulus $M_{(v, k)}$ map, the formulation of the saliency of haptic stimuli is also modulated by the current objectives of the exploration task. The objectives of the task being executed by the robotic platform are represented by the discrete random variable $T=$ "Task objective.", given that $T \in\left\{T_{1}, \ldots, T_{\Phi}\right\}$. During an experimental run the value of $T=t$ is considered constant throughout. $\Phi$ expresses the total number of different tasks that can be executed by the robotic platform.

Based on these considerations, the saliency of the haptic stimulus at $v$ is denoted by continuous random variable $S_{(v, k)}$, and is dependent on the class of tasks $T=$ "Search and follow of discontinuities between regions of surfaces with Material $_{a}$ and Material $_{b}$.". $S_{(v, k)}$ is related by a soft evidence relation with the perceived haptic stimulus $M_{(v, k)}$ characterization of the workspace (detailed description in Ref. [3]) given by

$S_{(v, k)}=\frac{\max \left(\left|s_{x}\right|,\left|s_{y}\right|,\left|s_{z}\right|\right)}{s_{n o r m}}, \quad S_{(v, k)} \in[0,1]$

The parameters $s_{x}=\mathcal{G}_{\text {sobel } l_{x}}(\mathbf{d}), s_{y}=\mathcal{G}_{\text {sobely }}(\mathbf{d})$ and $s_{z}=\mathcal{G}_{\text {sobel }}(\mathbf{d})$ are determined using a $3 \times 3$ edge detector $\mathbf{G}_{\text {sobel }}(3 \times 3$ kernel around $v)$ following an approach described in Refs. [3,31]. High values of $S_{(v, k)}$ correspond to regions around $v$ expressing a haptic discontinuity between Material $_{a}$ and Material $_{b}$.

\subsection{Inference of the next exploration target}

Based on the conditional independence relations between random variables $O_{k}, I_{(v, k)}, U_{(v, k)}, R_{k} S_{(v, k)}, T$, presented in Fig. 7 a), the joint probability distribution function $P\left(O_{k}, T, S_{(v, k)}, U_{(v, k)}, I_{(v, k)}, R_{k} \mid \pi_{t a r}\right)$ for this model $\pi_{t a r}$, is decomposed as summarised in Fig. 7b), including parametric forms corresponding to each factor. The final estimate for the next exploration target $\widehat{o}_{k}$ is given using a Maximum a Posteriori (MAP) decision rule, given a specific task $T=t$, as follows

$$
\begin{aligned}
& \widehat{o}_{k}=\underset{o_{k}}{\arg \max } P\left(O_{k} \mid t, s_{(v, k)}, i_{(v, k)}, u_{(v, k)}, R_{k},\right. \\
& \left.\pi_{t a r}\right) \Leftrightarrow \hat{o}_{k}=\underset{o_{k}}{\arg \max } \sum_{R_{k}}\left(\begin{array}{c}
P\left(t \mid \pi_{\text {tar }}\right) . P\left(i_{(v, k)} \mid O_{k}, \pi_{\text {tar }}\right) \cdot P\left(O_{k} \mid \pi_{\text {tar }}\right) \cdot \\
P\left(s_{(v, k)} \mid O_{k}, t, \pi_{t a r}\right) \cdot P\left(u_{(v, k)} \mid O_{k}, \pi_{t a r}\right) . \\
P\left(R_{k} \mid O_{k}, \pi_{t a r}\right)
\end{array}\right)
\end{aligned}
$$

The determination of the probability distribution functions $P\left(S_{(v, k)} \mid O_{k}, T, \pi_{t a r}\right), P\left(I_{(v, k)} \mid O_{k}, \pi_{t a r}\right), P\left(U_{(v, k)} \mid O_{k}, \pi_{t a r}\right), P\left(R_{k} \mid O_{k}, \pi_{t a r}\right)$ involved in Eq. (13) is described in detail next.

$$
\begin{aligned}
& \text { 5.3. Determination of } P\left(S_{(v, k)} \mid O_{k}, T, \pi_{t a r}\right), P\left(I_{(v, k)} \mid O_{k}, \pi_{t a r}\right) \text {, } \\
& P\left(U_{(v, k)} \mid O_{k}, \pi_{t a r}\right), P\left(R_{k} \mid O_{k}, \pi_{t a r}\right)
\end{aligned}
$$

As presented in Fig. $7 \mathrm{~b}), P\left(I_{(v, k)} \mid O_{k}, \pi_{t a r}\right)$ is described by a beta probability distribution function $\mathcal{B}_{I}$ characterised by the constants $\alpha_{I}=1$ and $\beta_{I}=2.5$. The profile of the probability distribution function

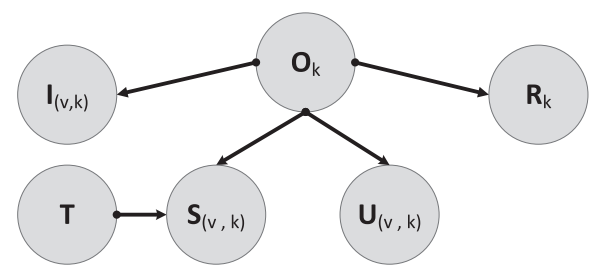

(a)

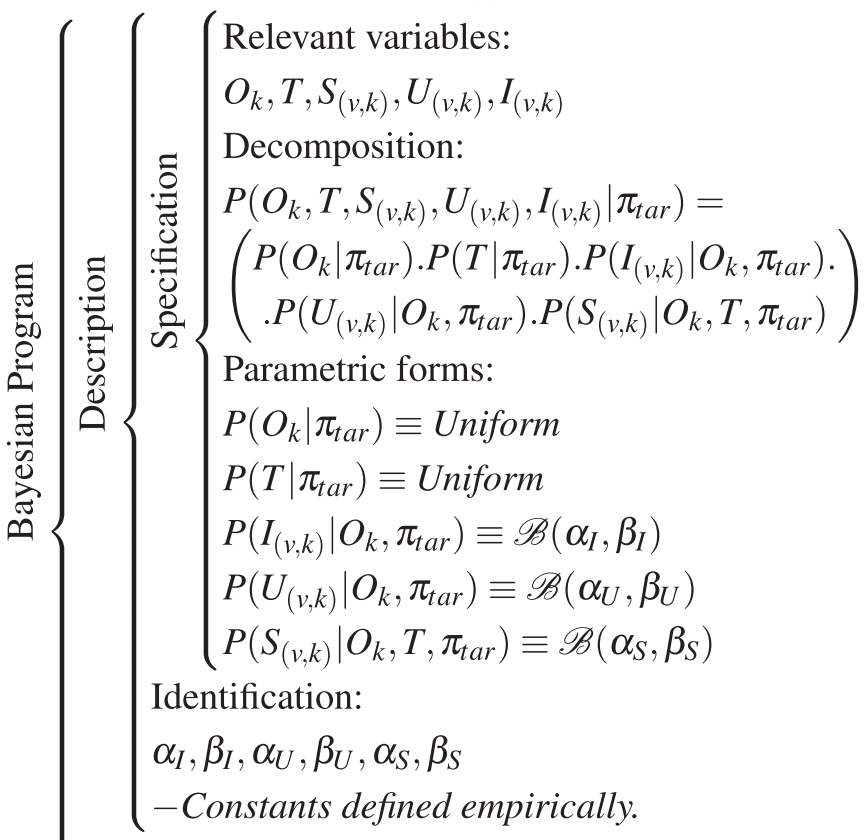

Question:

$P\left(O_{k} \mid t, s_{(v, k)}, u_{(v, k)}, i_{(v, k)}, \pi_{t a r}\right)$

Decision criteria: MAP - Maximum a posteriori.

$\hat{o}_{k}=\arg \max _{o_{k}} P\left(O_{k} \mid t, s_{(v, k)}, u_{(v, k)}, i_{(v, k)}, \pi_{t a r}\right)$

Fig. 7. Bayesianl model $\pi_{\text {tar }}$ :Selection of the next exploration target". a) Graphical representation. b) Description of the Bayesian program.

$P\left(I_{(v, k)} \mid O_{k}, \pi_{\text {tar }}\right)$ is represented in Fig. 8b). The selected profile for $P\left(I_{(v, k)} \mid O_{k}, \pi_{t a r}\right)$ attributes higher probabilities to lower levels of $I_{(v, k)}$ and lower probabilities to higher values of $I_{(v, k)}$ in order to promote the selection of regions of the workspace with low values of inhibition level.

Following an analogous approach, $P\left(U_{(v, k)} \mid O_{k}, \pi_{t a r}\right)$ is described by a beta probability distribution function $\mathcal{B}_{U}$ (Fig. 8b)) with the constant parameters $\alpha_{U}=4$ and $\beta_{U}=1 . P\left(U_{(v, k)} \mid O_{k}, \pi_{t a r}\right)$ attributes higher probability values to regions of the workspace perceived with higher uncertainty $U_{(v, k)}$, promoting the curiosity of the system.

$P\left(S_{(v, k)} \mid O_{k}, T, \pi_{t a r}\right)$ is described by a beta probability distribution function $\mathcal{B}_{R}$ defined by $\alpha_{R}=3$ and $\beta_{R}=1$ (Fig. 8 b)), assigning higher probability values to workspace regions $v$ with higher values of saliency $S_{(v, k)}$, promoting the exploration of regions of the workspace with relevant haptic stimulus for the task under execution.

The probability distribution function $P\left(R_{k} \mid O_{k}, \pi_{t a r}\right)$ is defined as a Gaussian Mixture Model (GMM), as follows

$P\left(R_{k}=\right.$ "Object $\left.{ }_{j} " \mid O_{k}, \pi_{\text {tar }}\right)=\sum_{i \in \text { template }_{j}^{\prime}} w_{i} \cdot g_{i}\left(O_{k} \mid \mu_{i}, \Sigma\right)$

The Gaussians $g_{i}$ of the GMM are centred at the locations $\mu_{i}$ of the workspace, with a covariance matrix $\Sigma$. Assuming a 2-D structure of the workspace, each Gaussian function $g_{i}$ is defined as follows: 


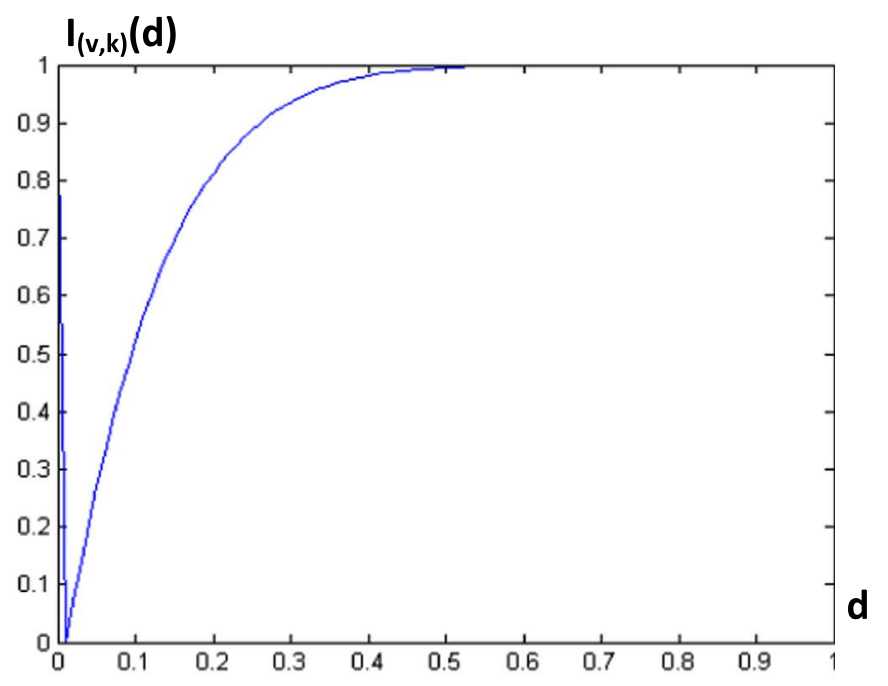

(a)

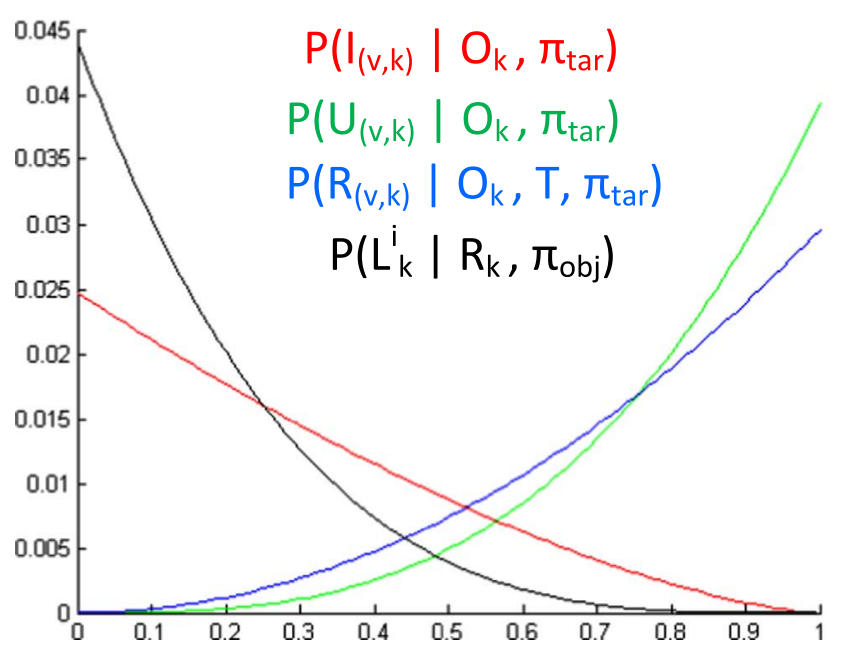

(b)

Fig. 8. Graphical representation of: a) $I_{(v, k)}$. b) $P\left(I_{(v, k)} \mid O_{k}, \pi_{t a r}\right), \quad P\left(U_{(v, k)} \mid O_{k}, \pi_{t a r}\right)$, $P\left(S_{(v, k)} \mid O_{k}, T, \pi_{t a r}\right), P\left(l_{k}^{i} \mid R_{k}, \pi_{o b j}\right)$.

$g_{i}\left(O_{k} \mid \mu_{i}, \Sigma\right)=\frac{1}{2 \pi^{(3 / 2)}|\Sigma|} \exp ^{-\frac{1}{2}\left(O_{k}-\mu_{i}\right) \Sigma^{-1}\left(O_{k}-\mu_{i}\right)}$.

The centers $\mu_{i}$ of the Gaussians correspond to the points belonging to the set Template $_{j}^{\prime}$, which are determined as presented in detail in Section 4.

\section{Experimental results}

\subsection{Computational simulation}

The path planning method proposed by this work, supporting the global haptic exploration strategy, was simulated computationally using MATLAB. The simulation scenario consists on a planar 2D probabilistic grid representing the workspace placed in front of a hypothetical robotic platform, as represented in Fig. 9. Three different materials were used: wood (Material ${ }_{10}$, brown cells), silicone (Material 8 , blue cells) and flush (Material 7 , green cells). The spatial distribution of the three materials intends to simulate an hypothetical real world scenario shown in Fig. 9. The workspace grid has the following lower and upper dimensions respectively $X_{l}^{W}=0 \mathrm{~m}$,
$X_{u}^{W}=0.30 \mathrm{~m}, Y_{l}^{W}=0 \mathrm{~m}, Y_{u}^{W}=0.60 \mathrm{~m}$. Each cell (square) has a side dimension of $\varepsilon=0.01 \mathrm{~m}$. This work considers that all the regions of the workspace are reachable by a robotic exploratory element.

As detailed previously in Section 1.2, this work does not address the low-level (motor and sensing) control loop involved in physical interaction between robotic fingers and surface. In the computational simulation, the sensory features modelling the haptic properties, texture $\left(E_{(v, k)}\right)$ and compliance $\left(C_{(v, k)}\right)$, of materials Material $_{7}$, Material $_{8}$, Material $_{10}$, were extracted from a previous work [10], as detailed in Section 3.

\subsection{Autonomous exploration of the workspace}

This work assumes that at each time iteration step $k$ of the system illustrated in Fig. 3, an exploratory element of a robotic hand probes a workspace region $v$. The sensory samples modelling texture $e_{(v, k)}$ and compliance $c_{(v, k)}$ are artificially synthesised from the respective probability distribution functions $P\left(E_{(v, k)} \mid m_{(v, k)}, \pi_{p e r}\right)$ and $P\left(C_{(v, k)} \mid m_{(v, k)}, \pi_{p e r}\right)$, given the known ground truth material $m_{(v, k)}$ for that region of the workspace, as defined in Fig. 9. Following the architecture of the sensory processing pipeline represented in Fig. 3, the sensory features samples $e_{(v, k)}, c_{(v, k)}$ are integrated by the Bayesian models to infer the next region (via point) of the workspace that should be probed by a robotic system.

In this scenario, the exploratory element of the robotic system initialized $(k=0)$ at random locations of the bi-dimensional grid representing the workspace region. The full-list of initialization locations for the 100 runs, is available online at http://www.rmartins.net/ j2016a. Unlike in previous work [3], these cells of the grid are not only located on a haptic discontinuity between the different materials of the scenario; they can be located on homogeneous regions. This provides a completely blind and unbiased initialization of the exploration task for each exploration run.

During each exploration task, the workspace presented in the Fig. 9 was explored during 100 runs (100 different initial locations of the exploratory element). For each run, the exploration procedures lasts 100 iterations (emulating time steps in realistic conditions) $k=0 \ldots 99$.

\subsubsection{Exploration tasks}

To evaluate the specificity and robustness of the Bayesian models implementing the touch attention mechanisms proposed in this work, the autonomous exploration of the workspace was performed using three different tasks $\left(T_{1}, T_{2}, T_{3}\right)$. The objectives are the following $T_{1}=$ "search and follow of discontinuities between Material $_{7}$ and remaining materials"; $T_{2}=$ "search and follow of discontinuities between Material $_{8}$ and remaining materials"; $T_{3}=$ "search and follow of discontinuities between Material $_{10}$ and remaining materials";.

\subsubsection{Performance metric}

Although the internal structure and configuration of the haptic stimulus disposed in the workspace is unknown a-priori to the robotic system, the ground truth describing the target locations (grid cells) of the workspace that should be probed by the robotic platform during task execution can be defined by a human operator for benchmarking purposes, and is denoted as $\mathcal{B}=\left\{\mathbf{b}_{1}, \mathbf{b}_{2}, \mathbf{b}_{3}, \ldots, \mathbf{b}_{l}\right\}, \mathbf{b}_{i}=(x, y) \in \mathbb{R}^{2}$. The set of workspace regions actually probed by the robotic platform during task execution, on the other hand, is denoted as $\mathcal{V}=\left\{\mathbf{v}_{1}, \mathbf{v}_{2}, \mathbf{v}_{3}, \ldots, \mathbf{v}_{k}\right\}$, $\mathbf{v}_{i}=(x, y) \in \mathbb{R}^{2}$.

The performance of the autonomous execution of the task by the robotic platform during an experimental run can be evaluated by the following error metric:

$\Gamma=\sum_{i=1}^{l}\left\|\mathbf{b}_{i}-\mathbf{v}_{\text {nearest }}\right\|, \quad$ given that $\forall_{\mathbf{v}_{i} \in \mathcal{V}} \quad \exists_{\mathbf{v}_{\text {nearest }}}$ :

$\left\|\mathbf{b}_{i}-\mathbf{v}_{\text {nearest }}\right\| \leq\left\|\mathbf{b}_{i}-\mathbf{v}_{i}\right\|$, 

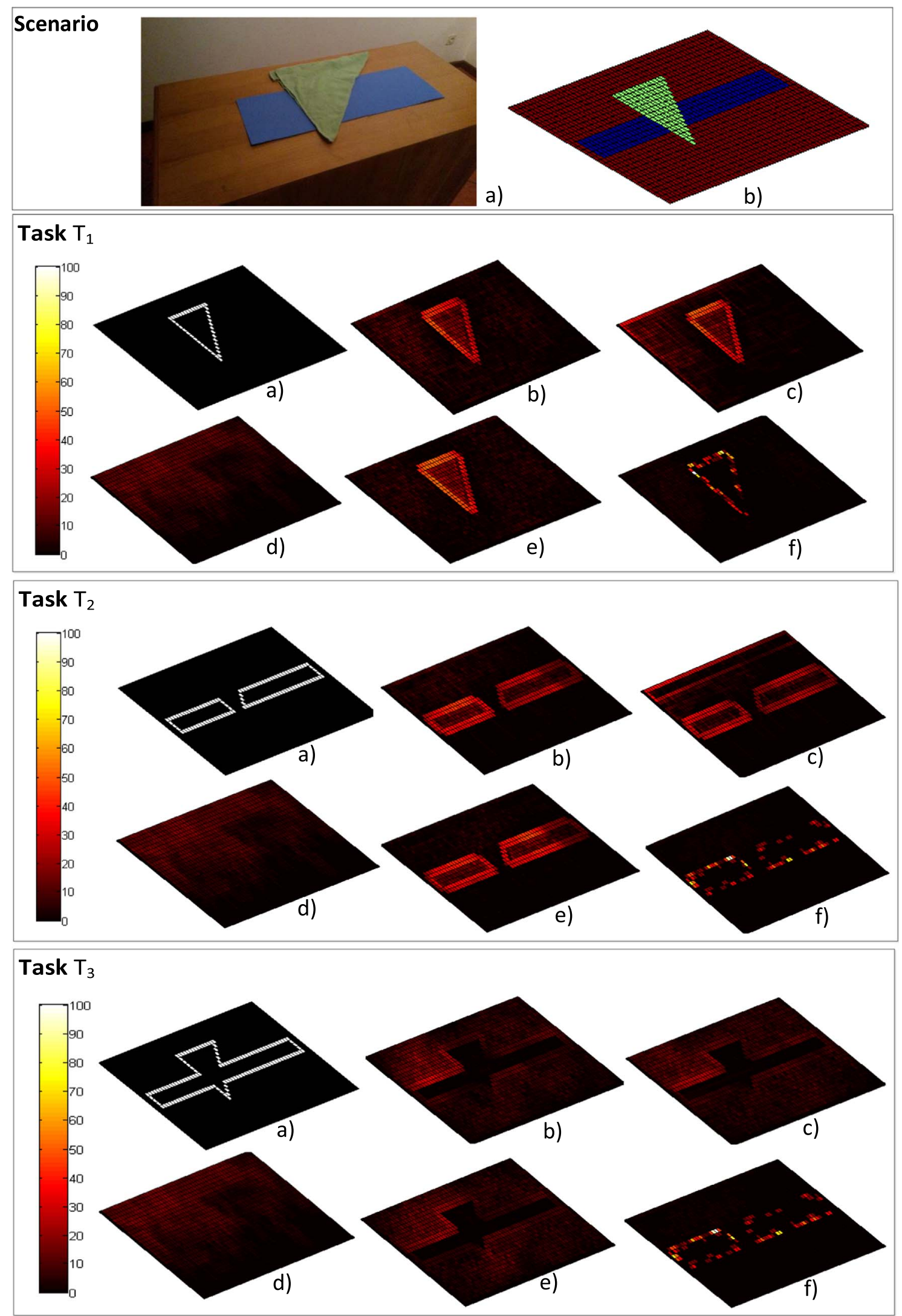

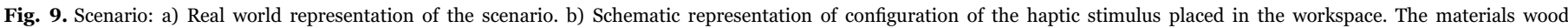

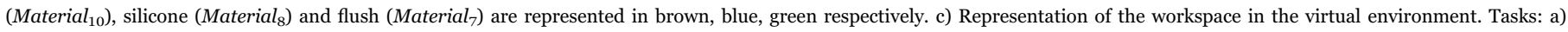

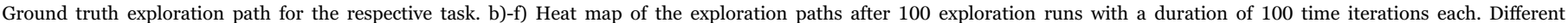

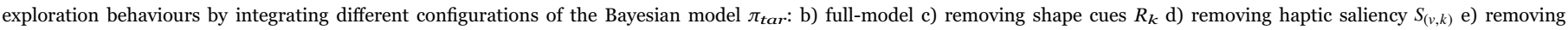

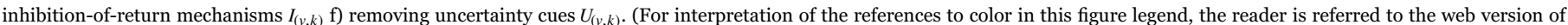
this article.) 
where $\|\ldots\|$ represents the Euclidean distance operator. This metric determines the total Euclidean distance between each ground truth point and the nearest point belonging the exploration path executed by the robotic platform. According to this approach, better autonomous exploration strategies provide lower values of $\Gamma$.

\subsection{Discussion of the experimental results}

The impact of the different components (discontinuity shape cues, uncertainty, haptic saliency, inhibition-of-return) of the Bayesian models implementing the touch attention mechanisms was evaluated by comparing the exploration performance after discarding specific components of the Bayesian model $\pi_{t a r}$ : shape cues $R_{k}$, haptic saliency $S_{(v, k)}$, inhibition-of-return mechanisms $I_{(v, k)}$, uncertainty cues $U_{(v, k)}$. The influence of those components was discarded by assuming that each of those random variables is described by a uniform probability distribution throughout the respective experimental runs.

Animated representations (time lapse) of the probability distribution functions during 100 time iterations involved in the progressive inference by the Bayesian model $\pi_{\text {tar }}$ of the workspace region that should be explored next, are available online at http://www.rmartins. net/j2016a, and an example is illustrated in Fig. 11.

The ground truth exploration paths for the objectives of the exploration tasks $T_{1}, T_{2}$ and $T_{3}$ are illustrated in Fig. 9 representing

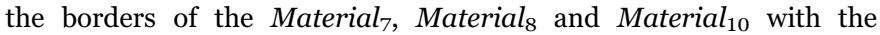
remaining materials in the workspace, respectively.

By performing a qualitative comparison between the ground truth exploration paths and the heat maps resulting from the exploration behaviour inferred by the full Bayesian model $\pi_{\text {tar }}$ in the Fig. 9, one finds that there is a high correspondence between the spatial structure of the most explored regions and the spatial structure of the ground truth exploration paths. The performance metric presented in Fig. 10 also shows that the full model always provides a good result. The touch attention mechanisms implemented by the Bayesian model $\pi_{\text {tar }}$ have promoted the exploration of regions corresponding to the discontinuities described in the objectives of the tasks $T_{1}, T_{2}, T_{3}$, ignoring other types of haptic discontinuities (Fig. 11).

The structural correspondence is higher for $T_{1}$ and $T_{2}$. This better performance is justified by the better perceptual discrimination capability of this system concerning Material $_{7}$ and Material $_{8}$ relatively to Material $_{10}$ (extensive study in Ref. [3]).

The analysis of the results of discarding the influence of specific components of the Bayesian model $\pi_{\text {tar }}$ (Fig. 9), shows that the degradation of performance of the exploration behaviour is significant (Fig. 10) when the effect of the haptic saliency $S_{(v, k)}$ is not considered. This causes the system to explore randomly the workspace, not taking into consideration any information about task relevance concerning the sensed haptic stimulus.

By neutralising the integration of the information about the uncertainty of the perceptual representation of the workspace (Fig. 9), the robotic system fails to have an exploration strategy that produces results similar to the ground truth. Although the Bayesian model $\pi_{\text {tar }}$ implements inhibition-of-return mechanisms, their effect is transient, and therefore, after some time elapses, the system tends to return to the same regions of the workspace that have been explored previously and were perceived with low uncertainty, thus providing a high saliency score for the task being executed. The plot of the performance metric $\Gamma$ for those conditions, shows that the degradation of performance of the exploration behaviour is considerable (Fig. 10).

By disabling the integration of the effects of the inhibition-of-return mechanisms, exploration task execution performance is less degraded. The plots of the $\Gamma$ metric, presented in Fig. 10, support this evidence by showing a performance of the system at the same level as the fullmodel condition. The removal of the transient effect of the inhibitionof-return mechanisms is compensated by the integration of information of mechanisms related with the uncertainty of the perceptual

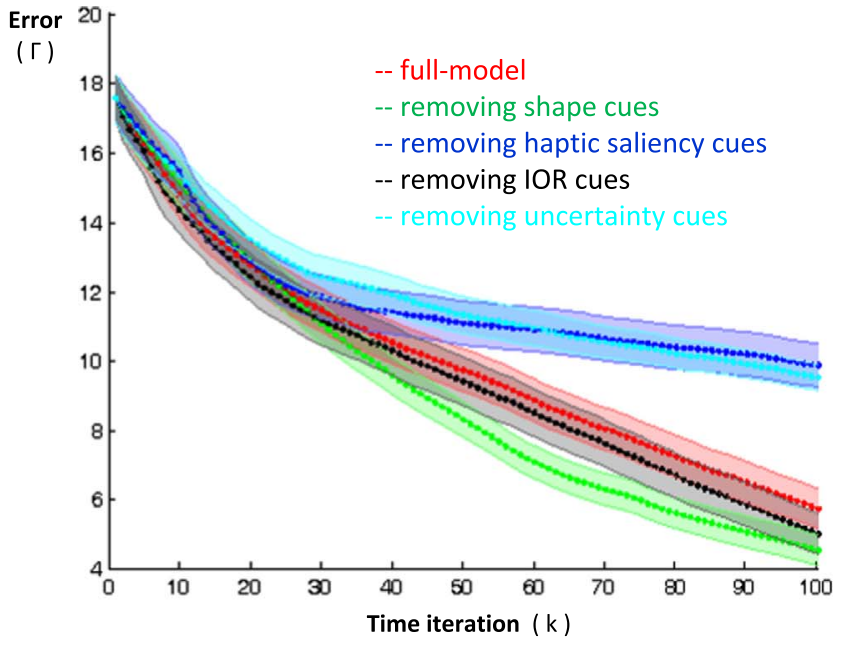

(a)

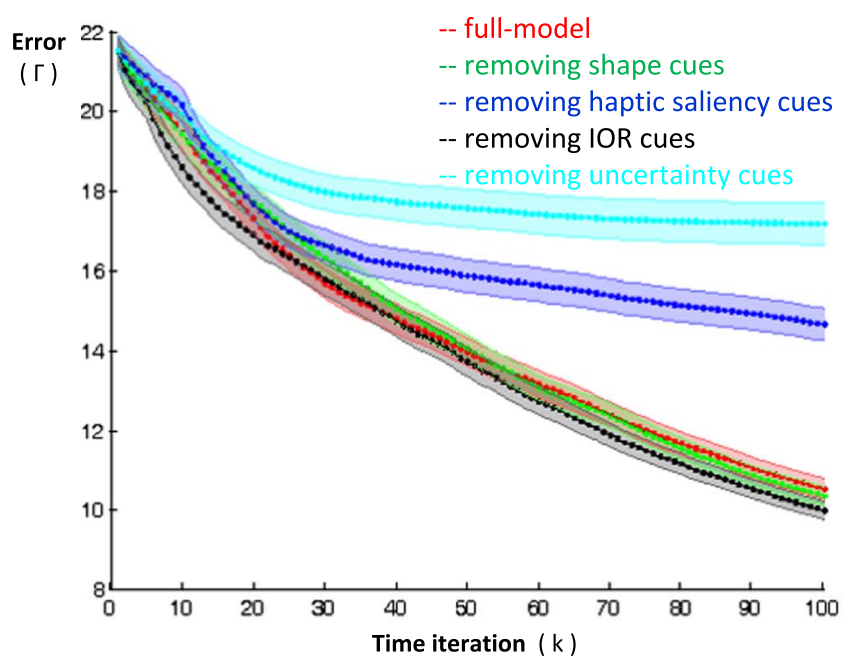

(b)

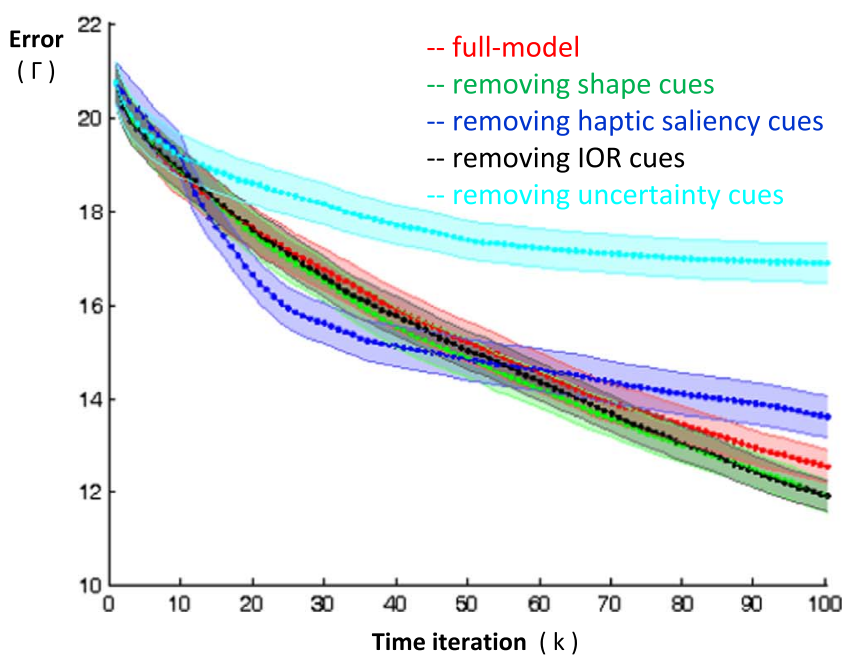

(c)

Fig. 10. Temporal evolution (from $k=0$ to $k=100$ ) of mean value (average for the 100 runs; shaded colors represent SEM: standard error of mean ) of performance metric $\Gamma$ by integrating different configurations of Bayesian model $\pi_{\text {tar }}$ : full-model, removing shape cues $R_{k}$, removing haptic saliency $S_{(v, k)}$, removing inhibition-of-return mechanisms $I_{(v, k)}$, removing uncertainty cues $U_{(v, k)}$. a) Task $T_{1}$. b) Task $T_{2}$. c) Task $T_{3}$. 

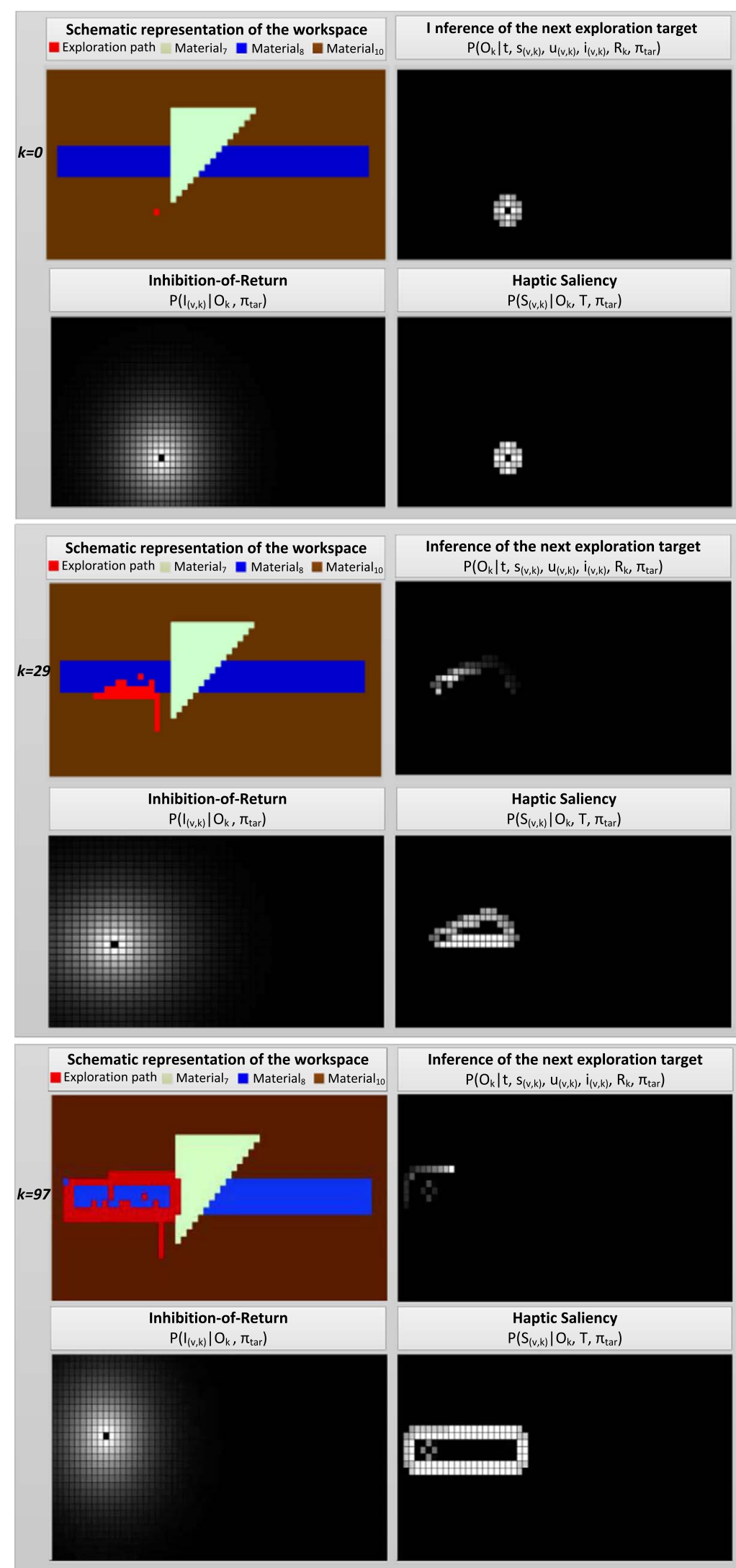

Haptic Saliency $\mathrm{P}\left(\mathrm{S}_{(\mathrm{v}, \mathrm{k})} \mid \mathrm{O}_{\mathrm{k}}, \mathrm{T}, \pi_{\mathrm{tar}}\right)$

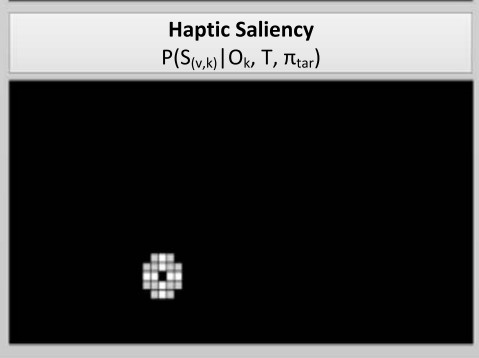

Inference of the next exploration target $P\left(O_{k} \mid t, s_{(v, k)}, u_{(v, k)}, i_{(v, k)}, R_{k}, \pi_{t a r}\right)$
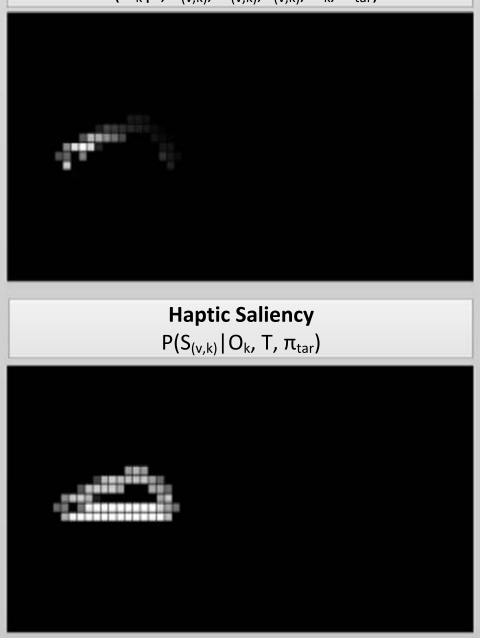

Inference of the next exploration target $P\left(O_{k} \mid t, s_{(v, k)}, u_{(v, k)}, i_{(v, k)}, R_{k}, \pi_{t a r}\right)$

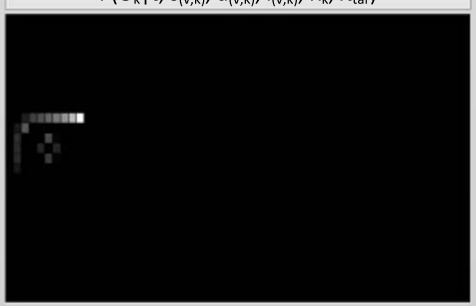

Haptic Saliency

$\mathrm{P}\left(\mathrm{S}_{(\mathrm{v}, \mathrm{k})} \mid \mathrm{O}_{\mathrm{k}}, \mathrm{T}, \pi_{\mathrm{tar}}\right)$

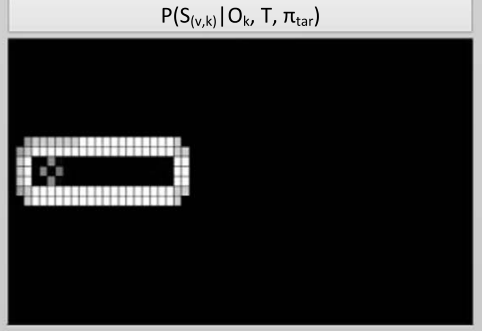

Shape similarity of exploration path $P\left(R_{k} \mid O_{k}, \pi_{t a r}\right)$
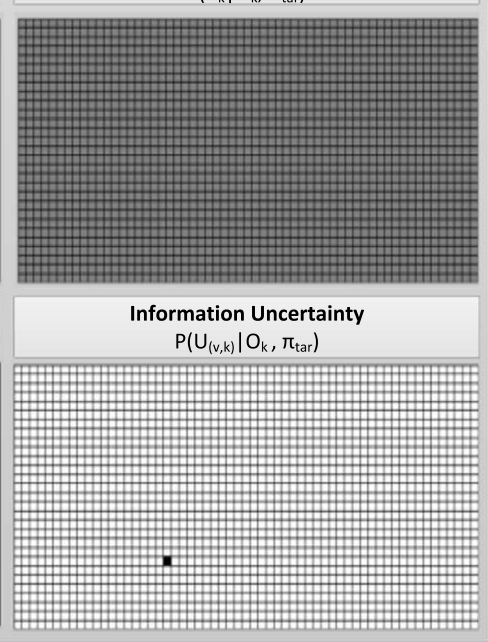

Shape similarity of exploration path $\mathrm{P}\left(\mathrm{R}_{\mathrm{k}} \mid \mathrm{O}_{\mathrm{k}}, \pi_{\mathrm{tar}}\right)$

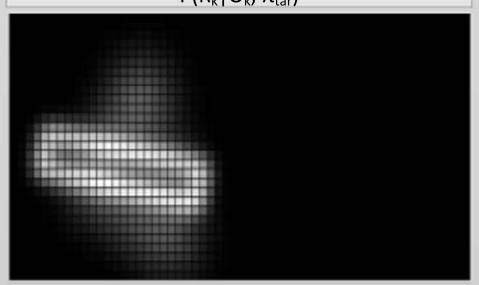

Information Uncertainty $\mathrm{P}\left(\mathrm{U}_{(\mathrm{v}, \mathrm{k})} \mid \mathrm{O}_{\mathrm{k}}, \pi_{\mathrm{tar}}\right)$

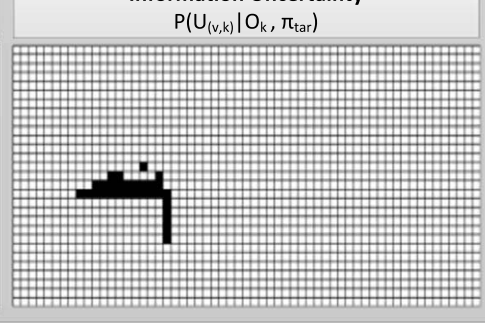

Shape similarity of exploration path $P\left(R_{k} \mid O_{k}, \pi_{t a r}\right)$

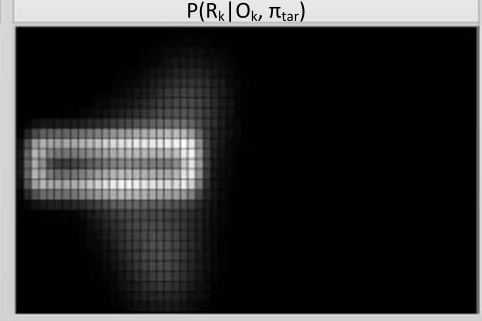

Information Uncertainty $\mathrm{P}\left(\mathrm{U}_{(\mathrm{v}, \mathrm{k})} \mid \mathrm{O}_{\mathrm{k}}, \pi_{\mathrm{tar}}\right)$

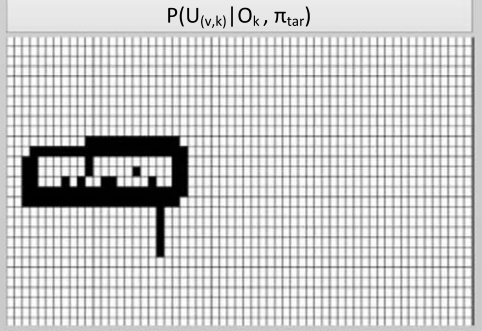

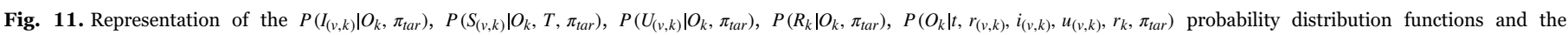

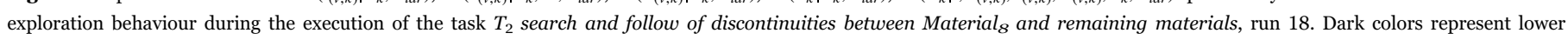

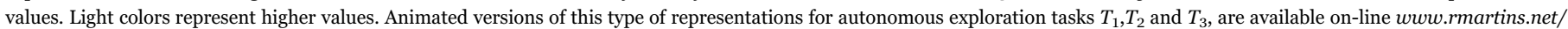
j2016a. 
Table 1

Summary of the relevant variables of this work.

\begin{tabular}{|c|c|c|}
\hline Variable & Description & Domain \\
\hline$v$ & Cell of the workspace grid. & $\mathbb{R}^{2}$ \\
\hline$k$ & Time / exploration iteration. & $\mathbb{N}_{0}$ \\
\hline$R_{k}$ & $\begin{array}{l}\text { Category of the structure of the } \\
\text { discontinuity. }\end{array}$ & $\left\{\right.$ "Shape,", "Shape ${ }_{2}$ "\} \\
\hline template $_{i}$ & $\begin{array}{l}\text { Set of points defining the template of each } \\
\text { category of structure. }\end{array}$ & $\mathbb{R}^{2}$ \\
\hline$L_{k}^{i}$ & $\begin{array}{l}\text { Matching error between the exploration } \\
\text { path and template } e_{i} .\end{array}$ & {$[0,1]$} \\
\hline$M_{(v, k)}$ & Material category of $v$ & $\left\{\right.$ Material $_{1}, \ldots$, Material $\left._{10}\right\}$ \\
\hline$E_{(v, k)}$ & Texture characterization of $v$. & $\mathbb{R}$ \\
\hline$C_{(v, k)}$ & Compliance characterization of $v$. & $\mathbb{R}$ \\
\hline $\mathbf{h}_{(v, k)}$ & Raw haptic sensing data acquired on $v$. & $\mathbb{R}^{n *}$ \\
\hline$O_{k}$ & Next workspace region to be explored. & $v$ \\
\hline$I_{(v, k)}$ & Inhibition level for cell $v$. & {$[0,1]$} \\
\hline$U_{(v, k)}$ & Uncertainty level for cell $v$. & {$[0,1]$} \\
\hline$S_{(v, k)}$ & $\begin{array}{l}\text { Saliency of the perceived haptic stimulus } \\
\text { in region } v \text {. }\end{array}$ & {$[0,1]$} \\
\hline$T$ & Objective of the haptic exploration task. & $\left\{T_{1}, T_{2}, T_{3}\right\}$ \\
\hline
\end{tabular}

representation of the workspace, which naturally correspond to less explored regions, if all surfaces in the workspace remain static/rigid. These regions tend to be avoided by the system, even without the influence of the inhibition-of-return mechanisms. The inhibition-ofreturn mechanisms may play a more relevant role in more ambiguous scenarios made of materials that the system can only perceive with high uncertainty, even after considerable exploration.

Discarding the effects provided by the integration of shape cues (Fig. 9), does not have a strong influence in the performance of the exploration behaviour of the system (Fig. 10). The weak contribution of the shape cues of the discontinuity to the improvement of the performance of the robotic system was caused by the low number of shape primitives recognised in this work (only two: rectangle and triangle) and by the high number of points that were used to describe each of the templates (around 50 points).

\section{Conclusions and future work}

The integration of touch attention mechanisms in the exploration of surfaces by robotic hands proved to be effective to search and follow haptic discontinuities based on noisy sensory data describing unknown scenes. The updated perceptual representation of the workspace, provided by the Bayesian model $\pi_{p e r}$, together with shape cues about the structure of the discontinuity being followed provided by the Bayesian model $\pi_{o b j}$ (extension of previous work [3]), are integrated by the Bayesian model $\pi_{\text {tar }}$ to perform perceptual inference and drive the decision process to determine the region that should be explored in the subsequent time step.

The Bayesian models were tested in a simulated scenario including three different materials during three different haptic exploration tasks. The results presented in Section 6.2, have demonstrated that the proposed approach provides the robotic system with a useful framework to define and generalise exploration behaviours. As in [22], the system was able to deal with severe changes in the slope of the discontinuities. In all the tasks, the robotic system was able to follow haptic discontinuities with progressive inversions in the slope of the discontinuity, what clearly demonstrates the generalization capability of the proposed approach. The emergent behaviour displayed by the system offers an improvement on the results presented in Ref. [22]. Testing the system with slope variations in discontinuities other than right angles (90 degrees) was suggested by [22] as a relevant future course of work. The touch attention mechanisms proposed in this work also showed high specificity. The robotic system followed the haptic discontinuities between the materials of interest for each task, ignoring other haptic discontinuities.

According with the results presented in Section 6.2, the performance of the robotic system during the haptic exploration tasks is heavily dependent on the integration by the Bayesian model $\pi_{\text {tar }}$ of information about the haptic saliency $S_{(v, k)}$ and uncertainty $U_{(v, k)}$ of the perceptual representation of the workspace. The formulation of the contributions of the inhibition-of-return mechanisms $I_{(v, k)}$ and shape cues of the haptic discontinuities $R_{k}$ is going to be studied extensively in future work, in order to improve and optimise the contributions of these components of the Bayesian model to the performance of the robotic system. In future developments of this work, elementary shape primitives should be recognised by the system and alternatives methods to ICP should be tested. This will allow the system to recognize earlier the tendencies in the shape of the discontinuity, matching the current exploration path with the shape templates more robustly (noise, scale, orientation). The future developments of this work will also investigate the implementation of the automatic computational optimization of the parameters defining the profile of Beta distribution functions. Currently, the selection of parameters is made empirically, testing different sets of values and analysing the behaviour of the system (Table 1).

In this work, the space used to formulate the solution of the global haptic exploration path planning consists in a $2 \mathrm{D}$ grid. The next developments of the proposed approach will study the extension of this space to a $3 \mathrm{D}$ grid. The operators and functions defined in 2D space during the formulation of the Bayesian models can be easily adjusted to 3D spaces (eg: exploration path matching with shape temples; Sobel operators involved in the formulation of haptic saliency; assignment of inhibition levels).

\section{Acknowledgements}

This research work was financially supported by an individual Ph.D. scholarship (SFRH/BD/65990/2009) funded by the Portuguese Science Agency FCT - Fundação para a Ciência e a Tecnologia (Foundation for Science and Technology).

\section{References}

[1] R.S. Johansson, J.R. Flanagan, Coding and use of tactile signals from the fingertips in object manipulation tasks, Nat. Rev. Neurosci. 10 (2009) 345-359.

[2] S.J. Lederman, The intelligent hand an experimental approach to human object recognition and implications for robotics and AI, AI Mag. 15 (1994) 774-785.

[3] R. Martins, J.F. Ferreira, J. Dias, Touch attention bayesian models for robotic active haptic exploration of heterogeneous surfaces, in: Proceedings of 2014 IEEE/ RSJ International Conference on Intelligent Robots and Systems (IROS 2014), IEEE, Chicago, USA, 2014, pp. 1208-1215. http://dx.doi.org/10.1109/IROS.2014. 6942711 URL 〈http://rmartins.net/iros2014a).

[4] F. Meng, B. Guo, M. Song, X. Zhang, Image fusion with saliency map and interest points, Neurocomputing 177 (2016) 1-8.

[5] S.-W. Ban, M. Lee, Selective attention-based novelty scene detection in dynamic environments, Neurocomputing 69 (1315) (2006) 1723-1727 [blind Source Separation and Independent Component AnalysisSelected papers from the ICA] [ 2004 meeting, Granada, SpainBlind Source Separation and Independent Component Analysis].

[6] R.B. Gomes, B.M. de Carvalho, L.M.G. Gonalves, Visual attention guided features selection with foveated images, Neurocomputing 120 (2013) 34-44 [image Feature Detection and Description].

[7] M.O. Ernst, H.H. Bulthoff, Merging the senses into a robust percept, Trends Cogn Sci. 8 (2004) 162-169.

[8] E. Wacker, Tactile feature processing and attentional modulation in the human somatosensory system, [Ph.D. thesis] TU Berlin.

[9] J.F. Ferreira, J. Dias, Probabilistic Approaches to Robotic Perception, Vol. 91 of Springer Tracts in Advanced Robotics, Springer, 2014.

[10] D. Xu, G.E. Loeb, J.A. Fishel, Tactile identification of objects using bayesian exploration, in: ICRA 2013, 2013.

[11] A.M. Okamura, M.R. Cutkosky, Feature detection for haptic exploration with robotic fingers, Int. J. Robot. Res. 20 (12) (2001) 925-938.

[12] C.M. Oddo, M. Controzzi, L. Beccai, C. Cipriani, M.C. Carrozza, Roughness encoding for discrimination of surfaces in artificial active-touch, IEEE Trans. Robot. 27 (3) (2011) 522-533.

[13] J.A. Fishel, G.E. Loeb, Bayesian exploration for intelligent identification of textures, Frontiers Neurorobotics 6

[14] D. Chathuranga, V. Ho, S. Hirai, Investigation of a biomimetic fingertip's ability to 
discriminate fabrics based on surface textures, in: International Conference Advanced Intelligent Mechatronics, 2013, pp. 1667-1674. http://dx.doi.org/10. 1109/AIM.2013.6584336.

[15] R. Martins, D. Faria, J. Dias, Representation framework of perceived object softness characteristics for active robotic hand exploration, in: HRI2012, USA, 2012.

[16] H. Liu, X. Song, J. Bimbo, L. Seneviratne, K. Althoefer, Surface material recognition through haptic exploration using an intelligent contact sensing finger, in: IROS 2012, IEEE, 2012, pp. 52-57.

[17] F. Castelli, An integrated tactile-thermal robot sensor with capacitive tactile array, IEEE T. Ind. Appl. 38 (1) (2002) 85-90. http://dx.doi.org/10.1109/28.980361.

[18] H. Liu, D.P. Noonan, B.J. Challacombe, P. Dasgupta, L.D. Seneviratne, K. Althoefer, Rolling mechanical imaging for tissue abnormality localization during minimally invasive surgery, IEEE Trans. Biomed. Eng. 57 (2) (2010) 404-414.

[19] L.L. Bologna, J. Pinoteau, J.-B. Passot, J.A. Garrido, J. Vogel, E.R. Vidal, A. Arleo, A closed-loop neurobotic system for fine touch sensing, Journal of Neural Engineering 10 (4).

[20] Q. Li, C. Schurmann, R. Haschke, H. Ritter, A control framework for tactile servoing, in: Proceedings of RSS, 2013.

[21] J. Bohg, M. Johnson-Roberson, M. Bjoandrkman, D. Kragic, Strategies for multimodal scene exploration, in: Intelligent Robots and Systems (IROS), 2010 IEEE/ RSJ International Conference on, 2010, pp. $4509-4515$.

[22] U. Martinez-Hernandez, T. Dodd, L. Natale, G. Metta, T. Prescott, N. Lepora, Active contour following to explore object shape with robot touch, in: World Haptics 2013, 2013, pp. 341-346.

[23] L.D. Harmon, Automated tactile sensing, Int. J. Robot. Res. 1 (2) (1982) 3-32.

[24] R.L. Klatzky, S.J. Lederman, V.A. Metzger, Identifying objects by touch an expert system, Percept. Psychophys. 37 (4) (1985) 299-302.

[25] P. Dario, D. De Rossi, Tactile sensors and the gripping challenge increasing the performance of sensors over a wide range of force is a first step toward robotry that can hold and manipulate objects as humans do, IEEE Spectr. 22 (8) (1985) 46-53. http://dx.doi.org/10.1109/MSPEC.1985.6370785.

[26] H.R. Nicholls, M.H. Lee, A survey of robot tactile sensing technology, Int. J. Robot. Res. 8 (3) (1989) 3-30.

[27] R.S. Dahiya, G. Metta, M. Valle, G. Sandini, Tactile sensing - from humans to humanoids, IEEE Trans. Robot. 26 (1) (2010) 1-20.

[28] Z. Zhang, Iterative point matching for registration of free-form curves and surfaces, International journal of computer vision.

[29] J.F. Ferreira, J. Dias, Attentional mechanisms for socially interactive robots - a survey, IEEE Trans. Auton. Ment. Dev., Spec. Issue Behav. Underst. Dev. Robot. (2014) 1-18 [in press].

[30] C.E. Shannon, A mathematical theory of communication, SIGMOBILE Mob. Comput. Commun. Rev. 5 (1) (2001) 3-55.

[31] P. Bhattacharya, D. Wild, A new edge detector for gray volumetric data, Computers in Biology and Medicine, 26(4).

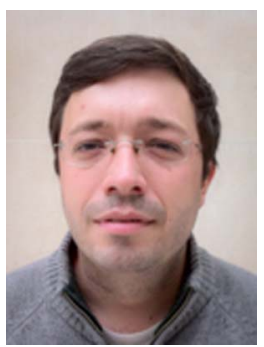

Ricardo Martins received his MSc degree in Biomedical Engineering from the University of Coimbra (UC) in 2008. Since 2010, Ricardo Martins is a PhD student at Institute of Systems and Robotics, UC. His PhD research interests are related with the modelling of the (haptic) artificial perception and action mechanisms involved in the dexterous robotic manipulation and exploration of objects and surfaces, inspired by the Human cognition and perception processes.

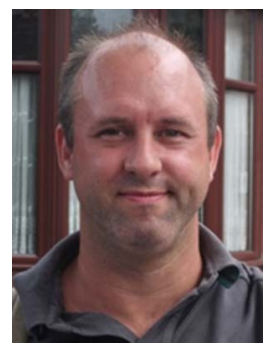

João Filipe Ferreira received the B.Sc., M.Sc., and Ph.D. degrees in electrical engineering and computers from the University of Coimbra (UC), in 2000, 2005, and 2011, respectively. He has been an Invited Assistant Professor at the UC since 2011. He has also been a researcher at the Institute of Systems and Robotics, UC, since 1999 (integrated member since 2011), where he is currently manager of the Artificial Perception for Intelligent Robots and Systems (AP4ISR) team. His main research interests are artificial cognition, probabilistic modelling, and autonomous systems, focusing in particular in human-robot interaction. Dr. Ferreira is a member of the IEEE Robotics and Automation Society (RAS) since 2012 (member of the IEEE RAS Technical Committee on Cognitive Robotics since 2015), the IEEE Life Sciences Community since 2013, the IEEE Systems, Man, and Cybernetics Society since 2015 and the IEEE Computational Intelligence Society since 2015.

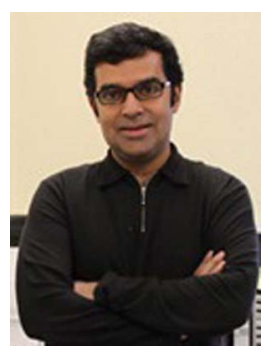

Miguel de Sá e Sousa Castelo-Branco received the M.D. degree from the University of Coimbra (UC), Portugal in 1991, and the Ph.D. degree from the Max-Planck Institute fur Hirn- forschung, Frankfurt, and the UC, in 1998. He is currently an Assistant Professor at the UC. He is also the Director of IBILI-UC (Institute for Biomedical Research on Light and Image) and ICNAS-UC (Institute of Nuclear Sciences Applied to Health). He has made contributions in the fields of ophthalmology, neurology, visual neuroscience, human psychophysics, functional brain imaging and human and animal neurophysiology.

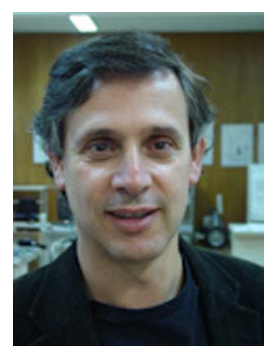

Jorge Dias received the Ph.D. degree in electrical engineering with specialization in control and instrumentation from the University of Coimbra (UC) in 1994. He holds his research activities at the Institute of Systems and Robotics (ISR), UC, and also at the Khalifa University of Science, Technology and Research , UAE. His current research areas are robot vision and autonomous robotics, with activities and contributions in these fields since 1984 . $\mathrm{He}$ has been the scientific coordinator in several projects supported by EU and by the Portuguese Foundation for Science and Technology (FCT). 